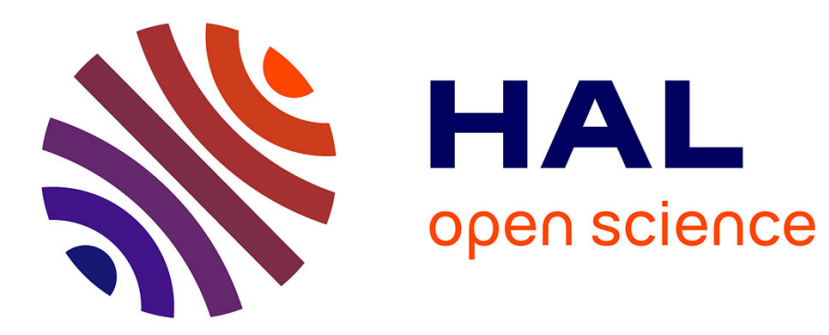

\title{
A novel multi-scale large deformation approach for modelling of granular collapse
}

Hao Xiong, Zhen-Yu Yin, François Nicot, Antoine Wautier, Marie Miot, Félix

Darve, Guillaume Veylon, Pierre Philippe

\section{- To cite this version:}

Hao Xiong, Zhen-Yu Yin, François Nicot, Antoine Wautier, Marie Miot, et al.. A novel multi-scale large deformation approach for modelling of granular collapse. Acta Geotechnica, 2021, 16, pp.23712388. 10.1007/s11440-020-01113-5 . hal-03120387

\section{HAL Id: hal-03120387 \\ https://hal-amu.archives-ouvertes.fr/hal-03120387}

Submitted on 25 Jan 2021

HAL is a multi-disciplinary open access archive for the deposit and dissemination of scientific research documents, whether they are published or not. The documents may come from teaching and research institutions in France or abroad, or from public or private research centers.
L'archive ouverte pluridisciplinaire HAL, est destinée au dépôt et à la diffusion de documents scientifiques de niveau recherche, publiés ou non, émanant des établissements d'enseignement et de recherche français ou étrangers, des laboratoires publics ou privés. 
Noname manuscript No.

(will be inserted by the editor)

\title{
A novel multi-scale large deformation approach for modelling of granular collapse
}

\author{
Hao Xiong · Zhen-Yu Yin* · François Nicot • Antoine \\ Wautier • Miot Marie • Félix Darve • Guillaume \\ Veylon - Pierre Philippe
}

Received: date / Accepted: date

\begin{abstract}
Collapse of granular material is usually accompanied by long run-out granular flows in natural hazards, e.g., rock/debris flow and snow avalanches. This paper presents a novel multi-scale approach for modelling granular column collapse with large deformation. This approach employs the smoothed particle hydrodynamics (SPH) method to solve large deformation boundary value problems while using a micromechanical model to derive the non-linear material response required by the SPH method. After examining the effect of initial cell size, the proposed approach is subsequently applied to simulate the flow of granular column in a rectangular channel at a low water content by varying the initial aspect ratio. The numerical results show good agreement with various experimental observations on both collapse process and final deposit morphology. Furthermore, the mesoscale behavior is also captured owing to the advantages of the micromechanical model. Finally, it was demonstrated that the novel multi-scale approach is helpful in improving the understanding of granular collapse and should be an effective computational tool for the analysis of real-scale granular flow.
\end{abstract}

Keywords Multiscale approach · Granular material · Micromechanics · Meso-scale Smoothed particle hydrodynamics $(\mathrm{SPH}) \cdot$ Granular collapse

* Corresponding author.

Hao Xiong

College of Civil and Transportation Engineering, Shenzhen University, Shenzhen, China.

E-mail: haoxiong19529@szu.edu.com

Zhen-Yu Yin

Department of Civil and Environmental Engineering, The Hong Kong Polytechnic University, Hung Hom, Kowloon, Hong Kong, P.R. China.

Tel.: +85234008470

Fax.:+852 23346389

E-mail: zhenyu.yin@polyu.edu.hk; zhenyu.yin@gmail.com

François Nicot

INRAE, Université Grenoble Alpes, UR ETGR, 2 rue de la Papeterie-BP 76, F-38402 St-Martin-d'Hères,France.

Antoine Wautier

INRAE, Aix-Marseille University, UR RECOVER, 3275 Rte Cézanne, CS 40061, F-13182 Aix-en-Provence Cedex 5, France

Miot Marie

INRAE, Aix-Marseille University, UR RECOVER, 3275 Rte Cézanne, CS 40061, F-13182 Aix-en-Provence Cedex 5, France

Félix Darve

Université Grenoble Alpes, CNRS, G-INP, Laboratoire 3SR UMR5521, Grenoble F-38000, France

Guillaume Veylon

INRAE, Aix-Marseille University, UR RECOVER, 3275 Rte Cézanne, CS 40061, F-13182 Aix-en-Provence Cedex 5, France

Pierre Philippe

INRAE, Aix-Marseille University, UR RECOVER, 3275 Rte Cézanne, CS 40061, F-13182 Aix-en-Provence Cedex 5, France 


\section{Introduction}

The transformation of granular material from a stationary state into a dynamic flow state can be a prototype found in many natural disasters and industrial processes. The understanding of granular flow mechanisms plays a crucial role in minimizing natural hazards (e.g., landslides, snow avalanches, and soil liquefaction) and in optimizing industrial processes (e.g., mineral processing, ceramic, food processing, pharmaceutical manufacture, and civil engineering applications). Many theories and assumptions have been proposed in the attempt to explain the apparent high mobility of granular material, including the bubbles at the bottom of granular flow (Shreve, 1968; Han et al., 2019), sand fluidization (Hungr and Evans, 2004; Jianbo et al., 2020), grain segregation-induced friction decrease (Phillips et al., 2006). However, these theories and assumptions have not been fully confirmed by experiments, and there are still many mechanisms of granular flow that are still unclear.

It has been recognized that debris flows and granular flows may behave similarly: for instance, they can sustain shear stresses with very slow deformation due to lasting, frictional grain contacts, and they can flow rapidly, sustaining inelastic grain collisions (Iverson, 1997; Fávero Neto et al., 2020). Thus, research has mainly focused on small scale laboratory experiments and numerical simulations of granular materials (Tan et al., 2019, 2020; Chen et al., 2019, 2020; Lajeunesse et al., 2005; Crosta et al., 2009; Lube et al., 2005, 2004; Rondon et al., 2011; Fern and Soga, 2017; Zhao et al., 2019; Brezzi et al., 2020; Kermani and Qiu, 2020). Among them, two classic experiments are widely performed: rectangular channel flowing tests and column flowing tests. In the former, granular collapse is obtained by putting the granular material in a rectangular channel and quickly removing a vertical side boundary, whereas, in the latter, granular material is in a hollow cylinder pipe. This study only discusses the former.

There are many possible solutions to simulate the granular column collapse by using numerical methods, e.g., the finite element method (FEM) using the arbitrary Lagrangian-Eulerian (ALE) technique (Crosta et al., 2009; Wu et al., 2019), the discrete element method (DEM) (Utili et al., 2015; Li et al., 2020), the particle finite element method (PFEM) (Zhang et al., 2015), the smoothing particle finite element method (SPFEM) (Jin et al., 2020), the material point method (MPM) (Sołowski and Sloan, 2015), and the smooth particle hydrodynamics (SPH) method (Peng et al., 2015; Fávero Neto and Borja, 2018; Wang et al., 2019; Peng et al., 2019; Bui et al., 2008; Yin et al., 2018; Jin et al., 2019b,a). Furthermore, some multi-scale approaches have also been employed to simulate the granular column collapse problem, e.g., a hierarchical coupling scheme to integrate MPM with DEM packings for multi-scale modelling of large deformation in granular materials (Liang and Zhao, 2019). These methods have contributed to the investigation of granular flows, but problems remain. On one side, although ALE technology can be used to solve large deformation problems in continuum methods, it does not really simulate the flow of granular materials because it lacks a unified formulation for going from solid to fluid in continuum mechanics. On the other side, the discretization methods require lots of calculations and are not suitable for large-scale boundary value problems. However, micromechanical models try to give the best compromise. The micromechanical models have no explicit constitutive behavior but the implicit description by statistical description of grain interactions, e.g. (Zhao et al., 2018; Zhu et al., 2006; Xiong et al., 2017; Nicot and Darve, 2011; Zhu et al., 2010; La Ragione et al., 2008; Zhao and Kruyt, 2020).

In this paper, a novel multi-scale framework is proposed. Under this framework, the SPH method is used to solve the large deformation boundary value problem while the $3 \mathrm{D}-\mathrm{H}$ model is used to derive the nonlinear constitutive material response of granular material required by $\mathrm{SPH}$ for each point. The 3D-H model is also extended to the condition of low water content by considering the capillary forces in water bridges between connected grains. Firstly, five simulations with different initial cell size are compared. Then, the proposed framework is subsequently applied to simulate the rectangular channel flowing tests at a low water content by varying the initial aspect ratio of the granular column. By comparing with various experimental observations, it verified that the proposed framework is able to predict the collapse process and the final deposit morphology. Furthermore, the mesoscopic analysis is performed owing to the advantages of the 3D-H model.

\section{Multi-scale framework for granular flows}

\subsection{Fundamentals of the SPH method}

The SPH method was adopted in this study to model large deformation of granular collapse. The SPH method was initially proposed by (Gingold and Monaghan, 1977; Lucy, 1977) in astrophysics. It was then introduced to other fields owing its further developments. The derivation of the SPH method can be divided into two stages. The first stage implements a kernel approximation, where all the physical variables 


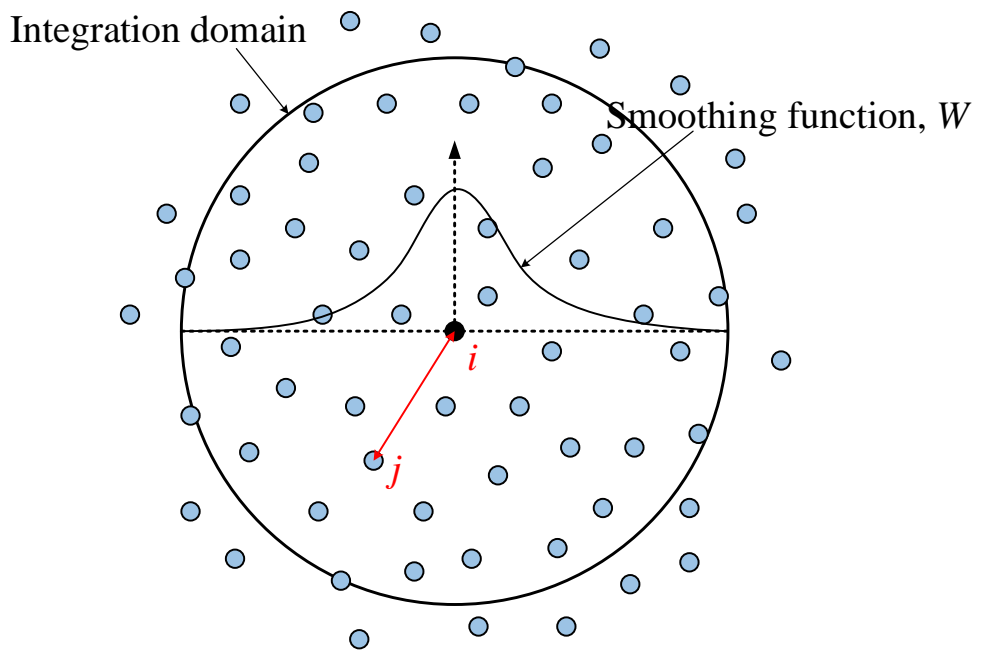

Fig. 1: Particle approximations using particles within the integration domain of the smoothing function $W$ for particle $i$.

are approximated using an integral transformation of discrete quantities involving the convolution of the physical variable with a chosen kernel function.

The kernel approximation of a function $f(x)$ at position $\boldsymbol{x}=(x, y, z)$ in physical domain $\Omega$ is expressed as

$$
\langle f(\boldsymbol{x})\rangle=\int_{\Omega} f\left(\boldsymbol{x}^{\prime}\right) W\left(\boldsymbol{x}-\boldsymbol{x}^{\prime}, h\right) d \boldsymbol{x}^{\prime}
$$

where $W$ is the kernel function and $h$ is the smoothing length.

The kernel function $W$ has to satisfy certain requirement such as normalization condition, delta function property and compact support condition (Monaghan, 1988; Peng et al., 2019). The cubic spine kernel is employed in this study. The kernel approximation of the gradient of the function $\nabla f(\boldsymbol{x})$ can also be obtained in the same manner to give

$$
\langle\nabla f(\boldsymbol{x})\rangle=-\int_{\Omega} f\left(\boldsymbol{x}^{\prime}\right) \nabla_{\boldsymbol{x}^{\prime}} W\left(\boldsymbol{x}-\boldsymbol{x}^{\prime}, h\right) d \boldsymbol{x}^{\prime}
$$

where $\nabla_{\boldsymbol{x}^{\prime}}$ indicates that the derivatives are evaluated at $\boldsymbol{x}^{\prime}$. If we assume that the continuous field $\langle f(\boldsymbol{x})\rangle$ comes from a piecewise constant field $f$, then the kernel representation for a function and its gradient as exhibited above can be approximated by a Riemann summation over the particles (i.e. the subdomains $\Omega_{j}$ where $f$ takes constant value $f\left(x_{j}\right), x_{j}$ being the center of $\left.\Omega_{i}\right)$ used in the simulation, thus

$$
\begin{gathered}
\langle f(\boldsymbol{x})\rangle=\sum_{j=1}^{N} f\left(\boldsymbol{x}_{j}\right) W\left(\boldsymbol{x}_{i}-\boldsymbol{x}_{j}, h\right) \frac{m_{j}}{\rho_{j}} \\
\langle\nabla f(\boldsymbol{x})\rangle=-\sum_{j=1}^{N} f\left(\boldsymbol{x}_{j}\right) \nabla_{j} W\left(\boldsymbol{x}_{i}-\boldsymbol{x}_{j}, h\right) \frac{m_{j}}{\rho_{j}}
\end{gathered}
$$

where $\rho_{j}$ and $m_{j}$ are the density and mass of particle $j\left(m_{j} / \rho_{j}=\left|\Omega_{j}\right|\right)$, respectively, and $N$ is the number of particles. Note that $\nabla_{i} W\left(\boldsymbol{x}_{i}-\boldsymbol{x}_{j}, h\right)=-\nabla_{j} W\left(\boldsymbol{x}_{i}-\boldsymbol{x}_{j}, h\right)$ due to the symmetry of the kernel function.

The second stage of the SPH method involves solving the equations governing granular impact; namely, the mass and momentum balance equations. These equations can be discretized with the kernel approximation as follows:

$$
\left\{\begin{array}{l}
\frac{\mathrm{d} \rho_{i}}{\mathrm{~d} t}=\sum_{j=1}^{N} m_{j}\left(v_{i}^{\alpha}-v_{j}^{\alpha}\right) \cdot \frac{\partial W\left(\boldsymbol{x}_{i}-\boldsymbol{x}_{j}, h\right)}{\partial x_{i}^{\alpha}} \\
\frac{\mathrm{d} v_{i}^{\alpha}}{\mathrm{d} t}=\sum_{j=1}^{N} m_{j}\left(\frac{\sigma_{i}^{\alpha \beta}+\sigma_{j}^{\alpha \beta}}{\rho_{i} \rho_{j}}+\Pi_{i j} \delta^{\alpha \beta}\right) \frac{\partial W\left(\boldsymbol{x}_{i}-\boldsymbol{x}_{j}, h\right)}{\partial x_{i}^{\beta}}
\end{array}\right.
$$




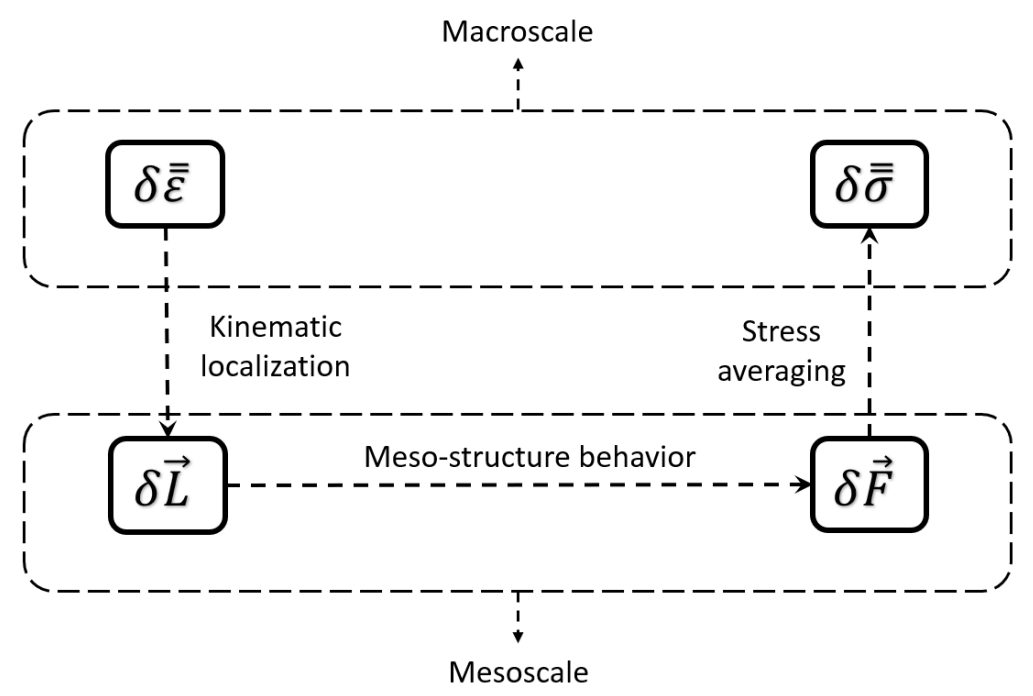

Fig. 2: General homogenization scheme of 3D-H model (Cambou et al., 1995).

where $\rho_{i}, v_{i}, p_{i}, \sigma_{i}, m_{i}$ are the density, velocity, pressure, stress and mass of particle $i ; \mathrm{d}(\cdot) / \mathrm{d} t$ denotes the time derivative of a physical quantity. The term $\Pi_{i j}$ in the momentum equation is the Monaghantype artificial viscosity. This term is introduced to smooth potential unphysical oscillations, to prevent unphysical particle-particle penetrations, and to stabilize the numerical solutions. The Monaghan-type artificial viscosity is incorporated into the momentum equation as follows (Monaghan, 1988):

$$
\Pi_{i j}= \begin{cases}\frac{-a c_{i j} \phi_{i j}+\beta \phi_{i j}^{2}}{\rho_{i j}} & \boldsymbol{v}_{\boldsymbol{i j}} \cdot \boldsymbol{x}_{\boldsymbol{i j}}<0 \\ 0 & \boldsymbol{v}_{\boldsymbol{i j}} \cdot \boldsymbol{x}_{\boldsymbol{i j}} \geq 0\end{cases}
$$

where $\phi_{i j}=\left(h_{i j} \boldsymbol{v}_{\boldsymbol{i j}} \cdot \boldsymbol{x}_{\boldsymbol{i j}}\right) /\left(\left|\boldsymbol{x}_{\boldsymbol{i j}}\right|^{2}+\varphi^{2}\right), c_{i j}=\frac{1}{2}\left(c_{i}+c_{j}\right), \rho_{i j}=\frac{1}{2}\left(\rho_{i}+\rho_{j}\right), h_{i j}=\frac{1}{2}\left(h_{i}+h_{j}\right), \boldsymbol{v}_{\boldsymbol{i j}}=\boldsymbol{v}_{\boldsymbol{i}}-\boldsymbol{v}_{\boldsymbol{j}}$, and $\boldsymbol{x}_{\boldsymbol{i} \boldsymbol{j}}=\boldsymbol{x}_{\boldsymbol{i}}-\boldsymbol{x}_{\boldsymbol{j}}$. Here $c_{\boldsymbol{i}}$ is the speed of sound associated with the particle $i ; \alpha$ and $\beta$ are constant coefficients that have values of about 1.0; and, $\phi=0.1 h_{i j}$ is applied in order to avoid singularity when two particles come too close.

\subsection{Multi-scale constitutive model: 3D-H model}

In Equation 5, the stress tensor has to be specified. This stress is linked to the strain field through a constitutive equation. In the present work, we chose to account for this constitutive equation in an implicit way with use of a micromechanical model. The 3D-H model (Xiong et al., 2017) was previously proposed by the authors and has been successfully implemented within a finite element code to solve geotechnical engineering problem (Xiong et al., 2019a,b). Based on the kinematic hypothesis, the 3D-H model enables the derivation of the macroscopic stress tensor from the macroscopic strain tensor according to the following steps (Figure 2):

(1) Kinematic localisation: The meso-structure (shown in Figure 4) is a connection between the macroand meso-scale. The dimension of the meso-structure can be characterised by the vector: $\boldsymbol{L}=\left[l_{1}, l_{2}, l_{3}\right]^{T}$, wherein $l_{1}, l_{2}, l_{3}$ represent the lengths along directions $\boldsymbol{n}, \boldsymbol{t}, \boldsymbol{w}$, respectively (see Figure 5a and Figure 6a). Thus the kinematic localisation assumption gives:

$$
\left\{\begin{array}{l}
\frac{\delta l_{1}}{l_{1}}={ }^{t} \boldsymbol{n} \cdot \boldsymbol{\delta} \boldsymbol{\varepsilon} \cdot \boldsymbol{n}=\left(\boldsymbol{P} \cdot \boldsymbol{\delta} \boldsymbol{\varepsilon} \cdot \boldsymbol{P}^{-1}\right)_{11} \\
\frac{\delta l_{2}}{l_{2}}={ }^{t} \boldsymbol{t} \cdot \boldsymbol{\delta} \boldsymbol{\varepsilon} \cdot \boldsymbol{t}=\left(\boldsymbol{P} \cdot \boldsymbol{\delta} \boldsymbol{\varepsilon} \cdot \boldsymbol{P}^{-1}\right)_{22} \\
\frac{\delta l_{3}}{l_{3}}={ }^{t} \boldsymbol{w} \cdot \boldsymbol{\delta} \boldsymbol{\varepsilon} \cdot \boldsymbol{w}=\left(\boldsymbol{P} \cdot \boldsymbol{\delta} \boldsymbol{\varepsilon} \cdot \boldsymbol{P}^{-1}\right)_{33}
\end{array}\right.
$$

where $\delta \varepsilon$ is the incremental macroscopic strain tensor, and $\boldsymbol{P}$ is the rotation matrix from global frame $\left(\boldsymbol{x}_{\mathbf{1}}, \boldsymbol{x}_{\mathbf{2}}, \boldsymbol{x}_{\mathbf{3}}\right)$ to local frame $(\boldsymbol{n}, \boldsymbol{t}, \boldsymbol{w})$ (see Figure 3 ).

The kinematic localisation defined by Equation 7 is a homogenisation process. It is analogous to the usual Voigt approximation in the field of continuous media. The reader should note that the localisation process goes from the macro- to the meso-scale, not to the micro-scale, as assumed in the Voigt approximation. 


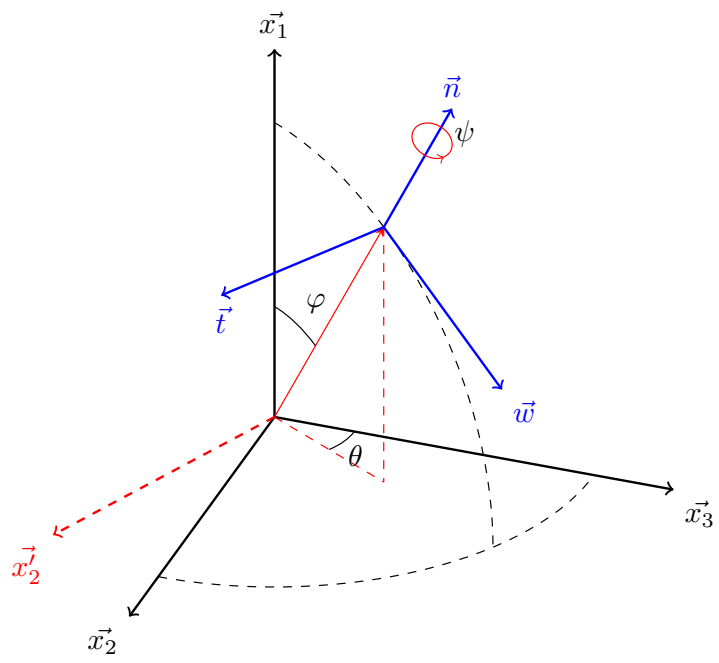

Fig. 3: Global and local coordinate system transformation by employing Euler angles in 3D conditions.

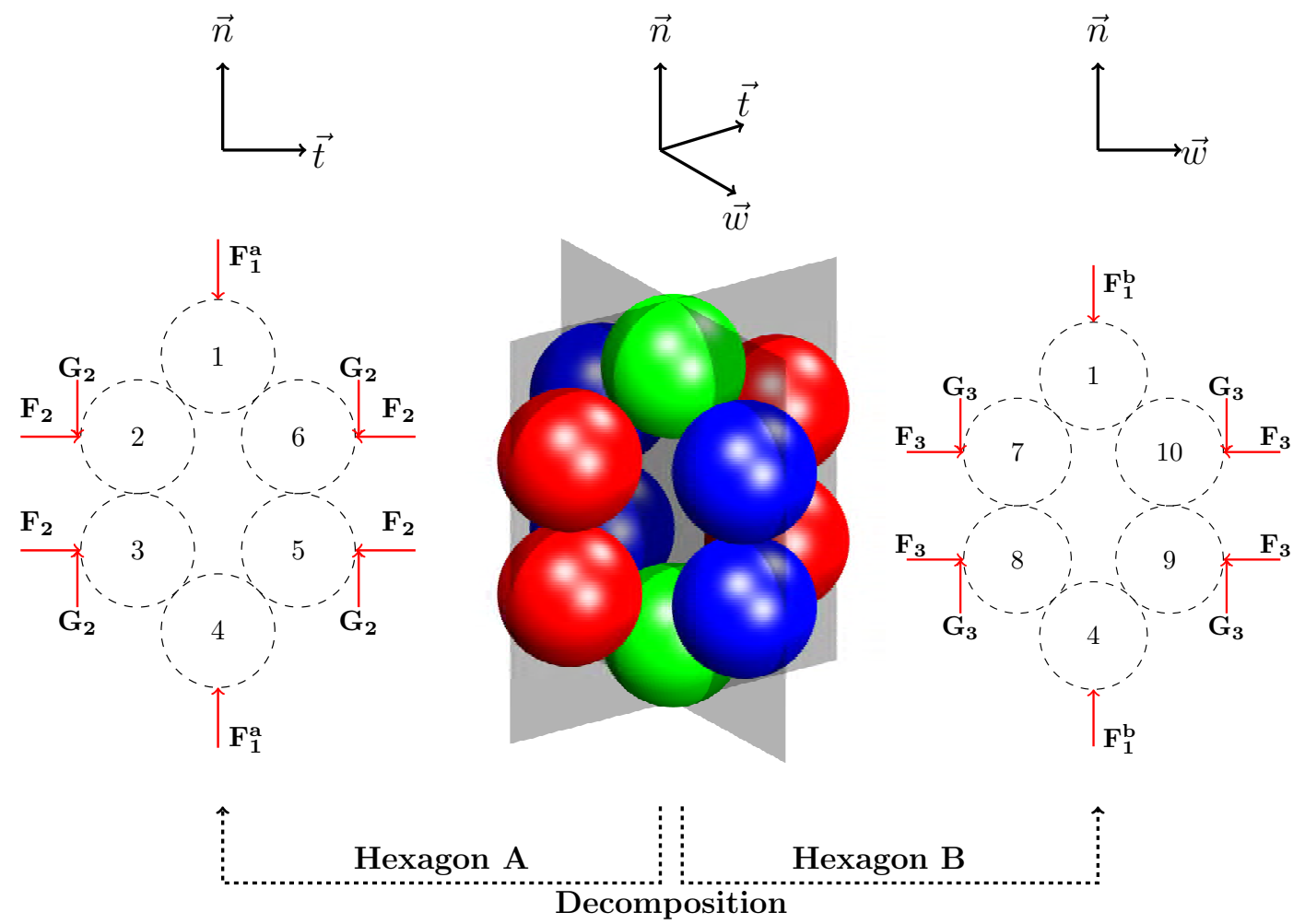

Fig. 4: The 3D mesostructure and its decomposition procedure in the 3D-H model.

Moreover, it has been widely used in granular materials, such as in (Cambou et al., 1995; Nicot and Darve, 2011; Nicot et al., 2005).

In the 3D-H model, the hexagon deformation described by the vector $\delta \boldsymbol{L}=\left[\delta l_{1}, \delta l_{2}, \delta l_{3}\right]^{T}$ is derived from the macroscopic strain tensor. Then, the term $\delta \boldsymbol{L}$ is used as the known variable to compute the relative displacement at each contact and to compute contact forces.

(2) Meso-structure behaviour: The meso-structure (Figure 4) can be decomposed into two independent hexagon patterns: Hexagon A (Figure 5) and Hexagon B (Figure 6), both being similar. The geometrical configuration and external forces applied to the meso-structure are symmetrical; thus for each hexagon, only two grains need to be analysed. For Hexagon A, as shown in Figure 5, only grains 1 and 2 are analysed. 
The contact between grains 1 and 2 is denoted by contact 1 , whereas the contact between grains 2 and 3 is denoted by contact 2 . Then, the kinematic relations read (for Hexagon A):

$$
\left\{\begin{array}{l}
\delta u_{n}^{1}=\delta d_{1} \\
\delta u_{t}^{1}=d_{1} \delta \alpha_{1} \\
\delta u_{n}^{2}=\delta d_{2}
\end{array}\right.
$$

where $u_{n}^{i}$ and $u_{t}^{i}$ represent the normal and tangential relative displacements at contact $i$ (For Hexagon A, $i=1$ or 2 , for Hexagon $\mathrm{B}, i=3$ or 4 ), respectively. As depicted in Figure 5 a, the geometrical description for Hexagon A gives:

$$
\left\{\begin{array}{l}
l_{1}=d_{2}+2 d_{1} \cos \alpha_{1} \\
l_{2}=2 d_{1} \sin \alpha_{1}
\end{array}\right.
$$

The force balance of grain 1 along direction $\boldsymbol{n}$ and of grain 2 along directions $\boldsymbol{w}$ and $\boldsymbol{n}$, together with the moment balance of grain 2, reads:

$$
\begin{aligned}
F_{1}^{a} & =2\left(N_{1} \cos \alpha_{1}+T_{1} \sin \alpha_{1}\right) \\
F_{2} & =N_{1} \sin \alpha_{1}-T_{1} \cos \alpha_{1} \\
N_{2} & =N_{1} \cos \alpha_{1}+T_{1} \sin \alpha_{1}+G_{2} \\
G_{2} & =T_{2}
\end{aligned}
$$

where $N_{i}$ and $T_{i}$ represent the normal and tangential contact forces of contact $i$, respectively. The elasticperfect plastic inter-particle contact law reads in an incremental form, for a given contact $i$ :

$$
\left\{\begin{array}{l}
\delta N_{i}=k_{n} \delta u_{n}^{i} \\
\delta \boldsymbol{T}_{\boldsymbol{i}}=\min \left\{\left\|\boldsymbol{T}_{\boldsymbol{i}}+k_{t} \delta \boldsymbol{u}_{\boldsymbol{t}}^{\boldsymbol{i}}\right\|, \tan \varphi_{g}\left(N_{i}+\delta N_{i}\right)\right\} \times \frac{\boldsymbol{T}_{\boldsymbol{i}}+k_{t} \delta \boldsymbol{u}_{\boldsymbol{t}}^{\boldsymbol{i}}}{\left\|\boldsymbol{T}_{\boldsymbol{i}}+k_{t} \delta \boldsymbol{u}_{\boldsymbol{t}}^{\boldsymbol{i}}\right\|}-\boldsymbol{T}_{\boldsymbol{i}}
\end{array}\right.
$$

After simplifying (see more details in Appendix A), the contact law (for Hexagon A) can be rewritten as follows:

$$
\left\{\begin{array}{l}
\delta N_{1}=-k_{n} \delta d_{1} \\
\delta N_{2}=-k_{n} \delta d_{2} \\
\delta T_{1}=B_{1} \delta \alpha_{1}-A_{1} \delta d_{1}+C_{1}
\end{array}\right.
$$

Term $C_{1}$ differs from zero only during a transition from an elastic to a plastic regime. For very small strain increments, as considered throughout this paper, term $C_{1}$ can therefore be neglected.

To obtain the incremental evolution of the external forces, $\delta d_{1}, \delta d_{2}$ and $\delta \alpha_{1}$ need to be expressed as a function of the mesoscopic strain. Three equations are therefore required. Compatibilities (Equations 9) provide two relations. The third is the balance equation of grain 2 along direction $\boldsymbol{n}$ (Equation 10c). By taking the inter-particle contact law (Equations 12) into account, we reach the following algebraic system expressing the incremental changes in $\delta d_{1}, \delta d_{2}$ and $\delta \alpha_{1}$ with respect to the incremental changes in $\delta l_{1}$ and $\delta l_{2}$ :

$$
\left[\begin{array}{ccc}
2 \cos \alpha_{1} & 1 & -2 d_{1} \sin \alpha_{1} \\
2 \sin \alpha_{1} & 0 & 2 d_{1} \cos \alpha_{1} \\
\cos \alpha_{1}+\frac{A_{1}}{k_{n}}\left(\sin \alpha_{1}+1\right) & -1 \frac{F_{2}-B_{1}\left(\sin \alpha_{1}+1\right)}{k_{n}}
\end{array}\right]\left[\begin{array}{c}
\delta d_{1} \\
\delta d_{2} \\
\delta \alpha_{1}
\end{array}\right]=\left[\begin{array}{c}
\delta l_{1} \\
\delta l_{2} \\
0
\end{array}\right]
$$

where $A_{1}, B_{1}$ are given in $\mathrm{A}$.

Differentiating Equation 10a, Equation 10b and combining with Equations 12 gives:

$$
\left\{\begin{array}{l}
\delta F_{1}^{a}=-k_{n} \cos \alpha_{1} \delta u_{n}^{1}+k_{t} \sin \alpha_{1} \delta u_{t}^{1}-F_{2} \delta \alpha_{1} \\
\delta F_{2}=-k_{t} \cos \alpha_{1} \delta u_{t}^{1}-k_{n} \sin \alpha_{1} \delta u_{n}^{1}+F_{1}^{a} \delta \alpha_{1}
\end{array}\right.
$$

Thus, combining Equations 8, Equations 13 and Equation 14, the incremental constitutive relation for Hexagon A can be expressed as follows:

$$
\frac{1}{|D|^{a}}\left[\begin{array}{ll}
K_{11}^{a} & K_{12}^{a} \\
K_{21}^{a} & K_{22}^{a}
\end{array}\right]\left[\begin{array}{l}
\delta l_{1} \\
\delta l_{2}
\end{array}\right]=\left[\begin{array}{l}
\delta F_{1}^{a} \\
\delta F_{2}
\end{array}\right]
$$




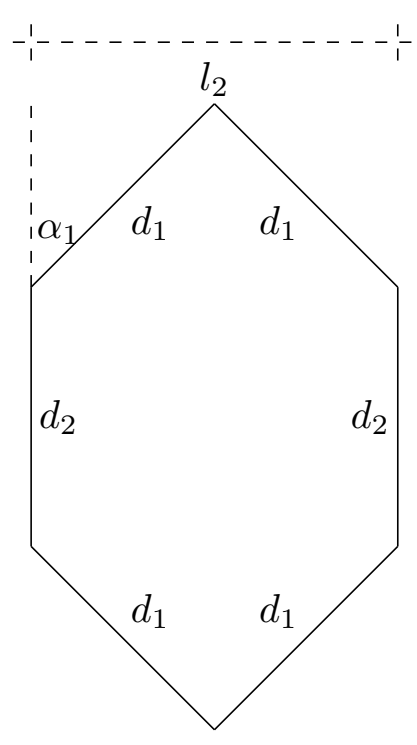

(a) Dimension of Hexagon A

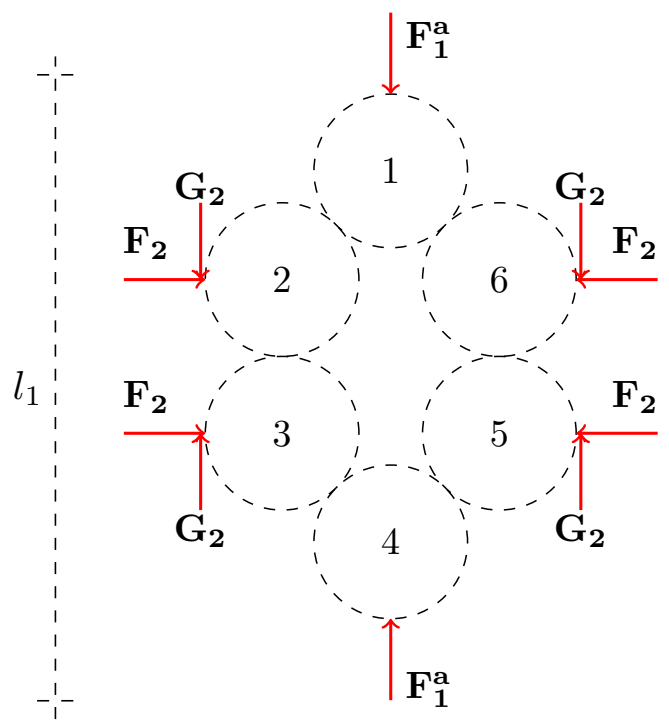

(b) External forces

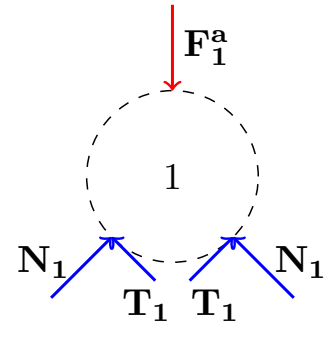

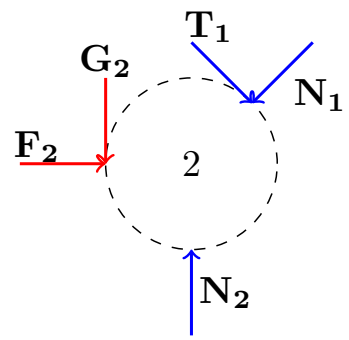

(c) Force balance for grains 1 and 2

Fig. 5: Mechanical description of Hexagon A.

where:

$$
\left\{\begin{aligned}
K_{11}^{a}= & 2\left(F_{2} \sin \alpha_{1}-k_{n} d_{1} \cos ^{2} \alpha_{1}-k_{t} d_{1} \sin ^{2} \alpha_{1}\right) \\
K_{12}^{a}= & \left(k_{t} d_{1} \sin \alpha_{1}-F_{2}\right)\left(\frac{A_{1}}{k_{n}} \sin \alpha_{1}+\frac{A_{1}}{k_{n}}+3 \cos \alpha_{1}\right) \\
& -\cos \alpha_{1}\left(B_{1} \sin \alpha_{1}+B_{1}-F_{2}+2 k_{n} d_{1} \sin \alpha_{1}\right) \\
K_{21}^{a}= & 2\left(k_{t}-k_{n}\right) d_{1} \sin \alpha_{1} \cos \alpha_{1}-2 F_{1}^{a} \sin \alpha_{1} \\
K_{22}^{a}= & \left(F_{1}^{a}-k_{t} d_{1} \cos \alpha_{1}\right)\left(\frac{A_{1}}{k_{n}} \sin \alpha_{1}+\frac{A_{1}}{k_{n}}+3 \cos \alpha_{1}\right) \\
& -\sin \alpha_{1}\left(B_{1} \sin \alpha_{1}+B_{1}-F_{2}+2 k_{n} d_{1} \sin \alpha_{1}\right) \\
|D|^{a}= & \frac{2}{k_{n}}\left(B_{1} \sin \alpha_{1}+A_{1} d_{1} \cos \alpha_{1}\right)\left(\sin \alpha_{1}+1\right) \\
& -\frac{2}{k_{n}}\left(F_{2} \sin \alpha_{1}+k_{n} d_{1} \cos ^{2} \alpha_{1}+2 k_{n} d_{1}\right)
\end{aligned}\right.
$$

Similarly, the incremental constitutive relation for Hexagon B can also be obtained. Consequently, superimposing Hexagon A and Hexagon B, the total incremental force along direction $\boldsymbol{n}$ is $\delta \boldsymbol{F}_{\mathbf{1}}=\delta \boldsymbol{F}_{1}^{\boldsymbol{a}}+\delta \boldsymbol{F}_{1}^{\boldsymbol{b}}$. The incremental constitutive relation of the 3D meso-structure is finally obtained.

(3) Stress averaging: Averaging the mesoscopic stress $\tilde{\boldsymbol{\sigma}}$ taking place within all the meso-structures in the specimen of volume $V$ can be performed as follows:

$$
\boldsymbol{\sigma}=\frac{1}{V} \iiint \omega(\theta, \varphi, \psi) \boldsymbol{P}^{-1} \widetilde{V} \widetilde{\boldsymbol{\sigma}}(\boldsymbol{n}, \boldsymbol{t}, \boldsymbol{w}) \boldsymbol{P} \sin \varphi d \theta d \varphi d \psi
$$

where $\boldsymbol{\sigma}$ is the macro-stress tensor operating on the specimen scale, $\widetilde{V}$ is the volume of meso-structure along the local frame $(\boldsymbol{n}, \boldsymbol{t}, \boldsymbol{w}), V$ is total volume of the specimen, which is computed from $\widetilde{V}$ as follows:

$$
V=\iiint \omega(\theta, \varphi, \psi) \widetilde{V} \sin \varphi d \theta d \varphi d \psi
$$

$\omega(\theta, \varphi, \psi)$ is a probability density function, which fulfills the following relation:

$$
\int_{0}^{2 \pi} \int_{0}^{\pi} \int^{2 \pi} \omega(\theta, \varphi, \psi) \sin \varphi d \theta d \varphi d \psi=1
$$




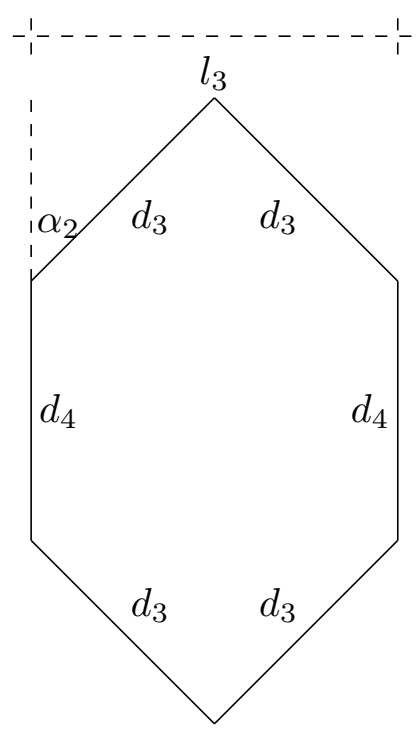

(a) Dimension of Hexagon B

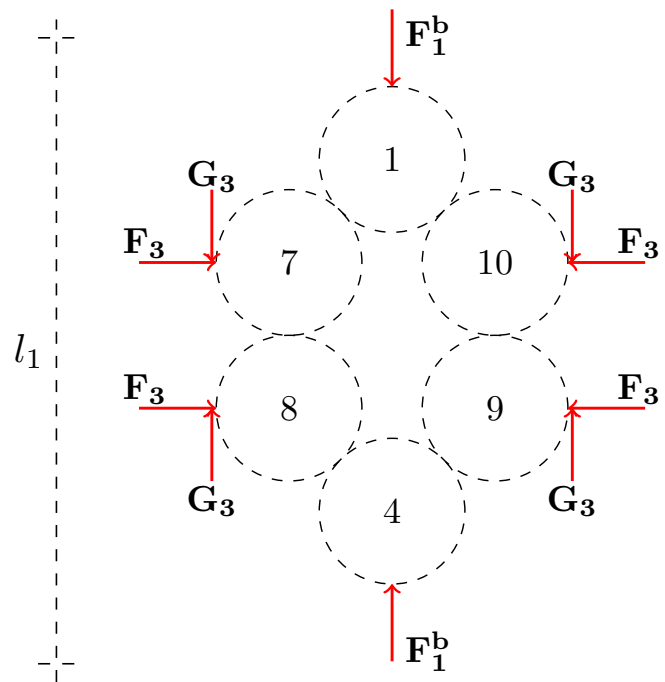

(b) External forces

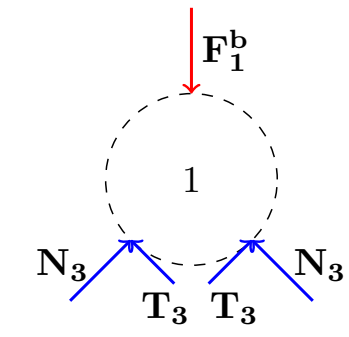

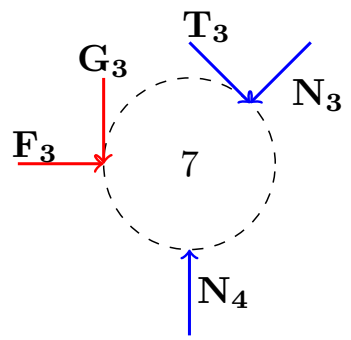

(c) Force balance for grains 1 and 7

Fig. 6: Mechanical description of Hexagon B.

For an isotropic specimen, $\omega_{0}=1 / 4 \pi^{2} . \tilde{V} \widetilde{\boldsymbol{\sigma}}$ with respect to the local frame can be computed from the local variables (Figure 5 and Figure 6) using the Love-Weber formula (De Saxcé et al., 2004; Love, 2013; Christoffersen et al., 1981; Mehrabadi et al., 1982):

$$
\left\{\begin{aligned}
\tilde{V} \widetilde{\sigma}_{11}= & 4 N_{1} d_{1} \cos ^{2} \alpha_{1}+4 T_{1} d_{1} \cos \alpha_{1} \sin \alpha_{1}+2 N_{2} d_{2} \\
& +4 N_{3} d_{3} \cos ^{2} \alpha_{2}+4 T_{3} d_{3} \cos \alpha_{2} \sin \alpha_{2}+2 N_{4} d_{4} \\
\widetilde{V} \widetilde{\sigma}_{22}= & 4 N_{1} d_{1} \sin ^{2} \alpha_{1}-4 T_{1} d_{1} \cos \alpha_{1} \sin \alpha_{1} \\
\widetilde{V} \widetilde{\sigma}_{33}= & 4 N_{3} d_{3} \sin ^{2} \alpha_{2}-4 T_{3} d_{3} \cos \alpha_{2} \sin \alpha_{2}
\end{aligned}\right.
$$

Notably, the local void ratio is related to the opening angle, which is not related to local anisotropy. The opening angle $\alpha_{1(2)}$ is a geometrical parameter (Figure 5 and Figure 6). The opening angle, together with the components $l_{1}, l_{2}, l_{3}$, determine the initial shape of the hexagons as well as the local void ratio of the meso-structure. For a virgin specimen, the initial opening angle is denoted as $\alpha_{0}$. Then, $\alpha_{1}=\alpha_{2}=\alpha_{0}$. The initial void ratio $e_{0}(\boldsymbol{n}, \boldsymbol{t}, \boldsymbol{w})$ of each meso-structure belonging to the local frame $(\boldsymbol{n}, \boldsymbol{t}, \boldsymbol{w})$ can be estimated using the initial opening angle $\alpha_{0}$ as follows:

$$
e_{0}=-\frac{4}{\pi} \cos ^{3} \alpha_{0}-\frac{6}{\pi} \cos ^{2} \alpha_{0}+\frac{4}{\pi} \cos \alpha_{0}+\frac{6}{\pi}-1
$$

In summary, the total number of input parameters of the 3D-H model is four (in case of initial microstructure isotropy). Three material parameters plus one geometry parameter. All parameters can be obtained by conventional drained triaxial test. The validations of this constitutive model have been conducted in our previous works (Xiong et al., 2017, 2019a, 2020), in which an excellent agreement between the 3D-H model and experimental data on dry sand has been achieved; therefore, these will not be repeated here.

\subsection{Extension of 3D-H model at a low water content}

At low water contents, water in a granular material forms individual capillary bridges between pairs of grains. These capillary bridges modify the constitutive behaviour of granular materials by introducing some cohesion. In this section, we show how the 3D-H model used so far can be extended to take into account capillary forces directly at contact scale for low water contents. The macroscopic effect of the capillary cohesion thus introduced is then investigated on the same numerical set up of Figure 13.

From a mechanical point of view, a capillary bridge between two grains can be taken into account in the form of an attractive force between the two connected grains. This capillary force can be computed with 
use of a surface energy minimization method. Thus, the total surface energy $E_{s}$ of two grains connected by a volume of water is given by

$$
E_{s}=\gamma^{l g} A^{l g}+\sum_{i=1}^{2} \gamma_{i}^{s l} A_{i}^{s l}+\sum_{i=1}^{2} \gamma_{i}^{s g} A_{i}^{s g}
$$

where $\gamma^{l g}=0.073 \mathrm{~N} . \mathrm{m}^{-1}$ is the surface tension of air/water interface at $20^{\circ} \mathrm{C}$ given in Molenkamp and Nazemi (2003), $A^{l g}$ is the area of the liquid/gas interface, $\gamma_{i}^{s l}$ and $A_{i}^{s l}$ are respectively the surface tension and the area of the interface between grain $i \in\{1,2\}$ and water, and $\gamma_{i}^{s g}$ and $A_{i}^{s g}$ are respectively the surface tension and the area of the interface between grain $i$ and air.

The surface tensions of the interfaces are related to the wetting angle $\theta$ by the Young-Dupré equation Yuan and Lee (2013):

$$
\gamma_{i}^{s l}-\gamma_{i}^{s g}=-\cos \theta_{i} \gamma^{l g}
$$

Thus the surface energy can be expressed by:

$$
E_{s}=\gamma^{l g} A^{l g}-\gamma^{l g} \sum_{i=1}^{2} \cos \theta_{i} A_{i}^{s l}+C
$$

where $C=\sum_{i=1}^{2} \gamma_{i}^{s g} A_{i}^{s}$ is a constant, with $A_{i}^{s}=A_{i}^{s g}+A_{i}^{s l}$ the total area of grain $i$.

The geometries of the liquid/gas and the solid/liquid interfaces are determined numerically with the surface energy minimization method implemented in Surface Evolver Brakke (1992). The numerical procedure is briefly detailed in the following. First, a rough mesh of the interfaces is created. With use of a gradient descent method, the nodes of the mesh are moved in order to minimize the total energy while keeping constant the volume of water. Then, the mesh is refined and the gradient descent procedure repeated until a precise geometry of the capillary bridge is obtained.

Eventually, the capillary force acting between the two grains is obtained from the virtual work principle. As presented in Figure 7, an incremental displacement $\delta d$ is imposed to one of the grain in the direction of the bridge, and the surface energy of this new configuration $E_{s}(\delta d)$ is calculated and compared with the original one. In a closed, static system without gravity, there are neither kinetic nor potential and internal energy. The incremental work $\delta W$ of the capillary force $F_{c}$ is thus related to the surface energy variation as:

$$
\delta W=\boldsymbol{F}_{\boldsymbol{c}} . \delta \boldsymbol{d}=\delta E_{s}
$$

This means that the capillary force $F_{c}$ can be expressed as a function of the variation of surface energy $E_{s}(\delta d)-E_{s}(0)$ and the incremental displacement $\delta d$ :

$$
F_{c}=\frac{E_{s}(\delta d)-E_{s}(0)}{\delta d}
$$

By repeating the whole procedure for varying liquid volume, the dimensionless capillary force $F_{c}^{*}=\frac{F_{c}}{\gamma^{s l} r}$ and the half-filling angle $\beta$ (as defined in Figure 7) can be computed as functions of the dimensionless liquid volume $V^{*}=V / R^{3}$ where $R$ is the grain radius. Note that, in the present work, the two grains are of equal radius and in contact. The reason for this restriction lies in the fact that the purpose of these simulations is to provide an empirical expression of the capillary force acting in between the contacting grains of the ten grain meso-structure of the H-model (Figure 10). Figure 8 shows that the capillary force in a liquid bridge can be approximated fairly well with a 4th degree polynomial while Figure 9 shows that the half filling angle can be approximated really accurately with a power law:

$$
\left\{\begin{array}{l}
F_{c}^{*}=0.701 V^{*^{4}}-3.167 V^{*^{3}}+5.150 V^{*^{2}}-4.290 V^{*}+5.380 \\
\beta=68.0 V^{*^{0.293}}
\end{array}\right.
$$

For low water contents, water in a meso-structure forms independent capillary bridges between grains two by two, as presented in Figure 10.

We suppose that the water content $w_{c}$ provided at macroscale is the same in all the meso-structures (whatever their local orientations). This hypothesis can be seen as a localisation of the water content, as for the strain localisation. Under this hypothesis, the dimensionless water volume $V_{w}^{*}$ in a given meso-domain is:

$$
V_{w}^{*}=w_{c} V_{s}^{*} \frac{\gamma_{s}}{\gamma_{w}}
$$




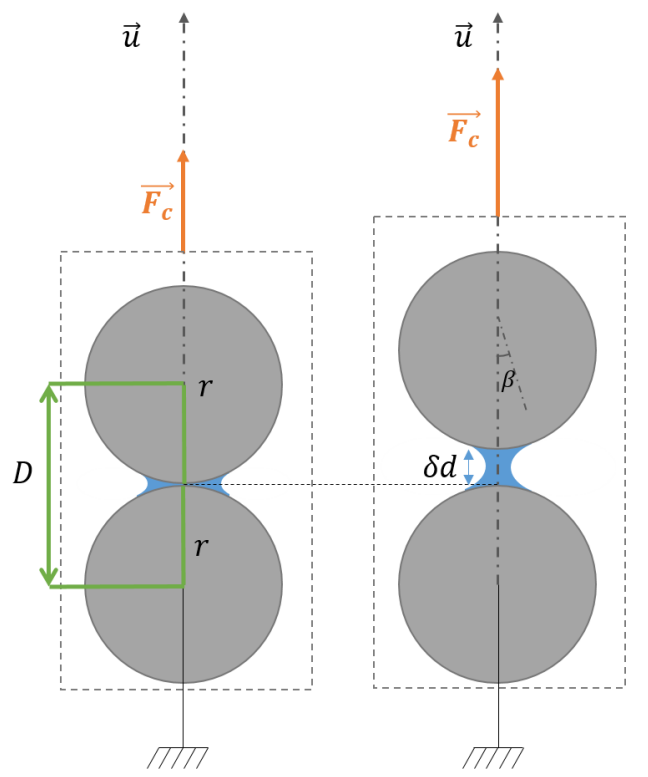

Fig. 7: Calculation of capillary force in a liquid bridge based on the Virtual Work Principle.

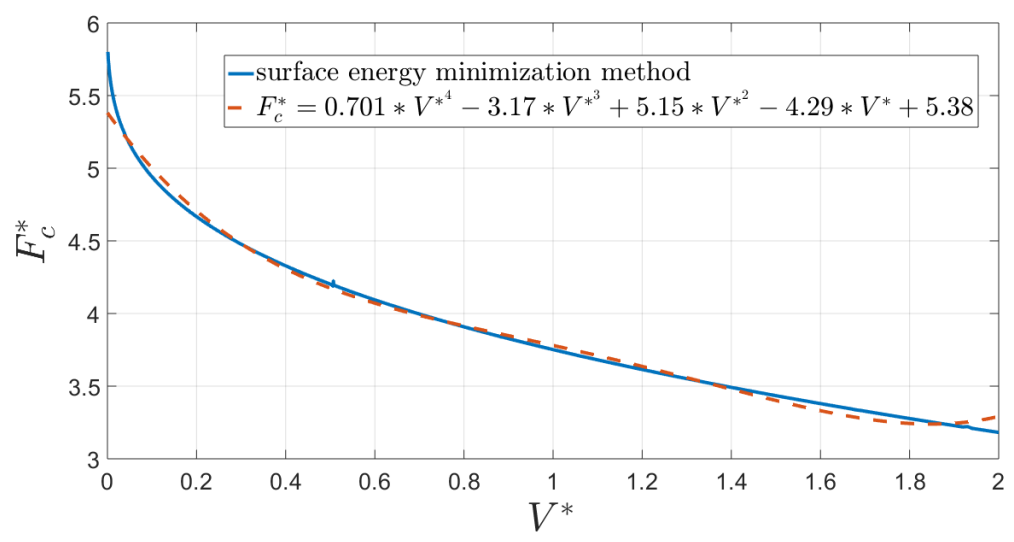

Fig. 8: Capillary force in a liquid bridge between two grains in contact as a function of the water volume calculated with the surface energy minimization method (solid line) and approached by a 4th degree polynomial (dashed line).

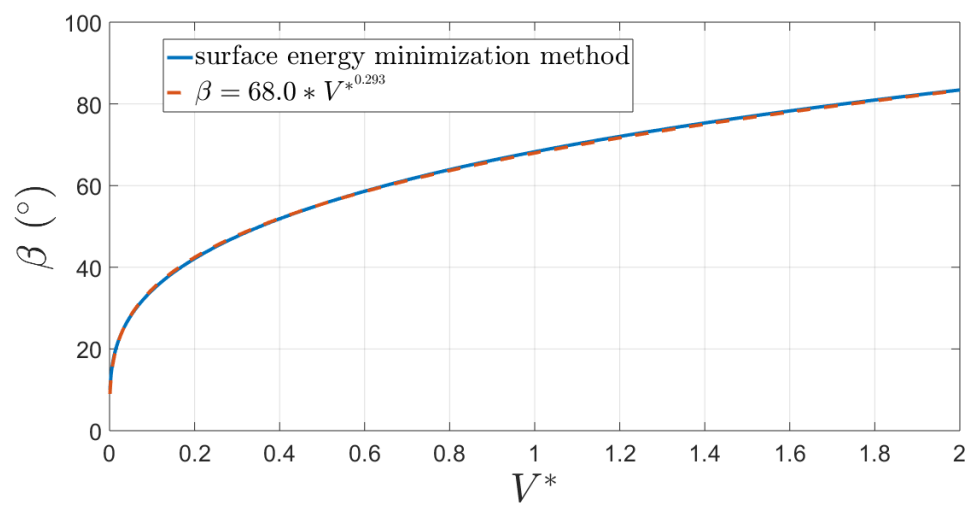

Fig. 9: Half-filling angle in a liquid bridge between two grains in contact as a function of the water volume calculated with the surface energy minimization method (solid line) and approached by a power law (dashed line). 


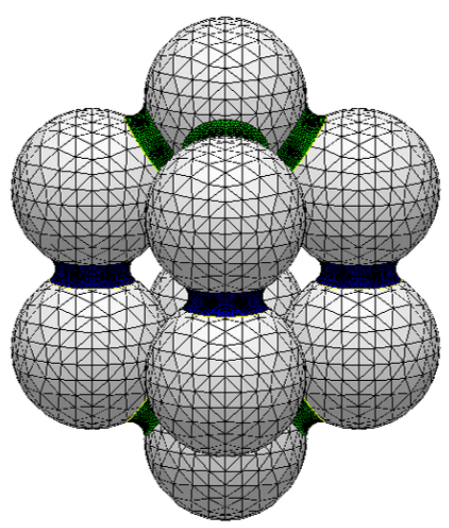

Fig. 10: Individual capillary bridges between grains in the H-model meso-structure. Vertical bridges are coloured in blue while inclined bridges are coloured in green

with $V_{s}$ being the volume of the 10 grains contained into the meso-domain $V_{s}^{*}=\frac{V_{s}}{r^{3}}=\frac{40}{3} \pi$, and $\frac{\gamma_{s}}{\gamma_{w}}=2.4$ the ratio of the solid and liquid specific weight.

The total volume of water $V_{w}^{*}$ lying inside the dodecahedron come from the contribution of the four vertical bridges and eight inclined bridges visible in Figure 10. To keep the symmetry of the meso-structure, we assume that all vertical bridges have the same volume $V_{v}^{*}$ and all inclined bridges have the same volume $V_{i}^{*}$. As a result, the total volume of water splits as:

$$
V_{w}^{*}=8 V_{i}^{*}+4 V_{v}^{*}
$$

In the above equation, we have one degree of freedom to split the volume $V_{w}^{*}$ between $V_{i}^{*}$ and $V_{v}^{*}$. We suppose that the distribution of water volume between the different capillary bridges is the distribution which minimizes the total surface energy in the meso-structure. However, this distribution has to insure that the bridges are not in contact, which would involve the merging of capillary bridges into more complex coalesced bridges (this particular point will be deeply investigated in a forthcoming paper). To carry on the computation, it is hypothesized that grain interpenetration is neglected so that the two hexagonal patterns of a cell are identical. Thus, it is assumed that:

$$
\left\{\begin{array}{l}
\alpha=\frac{\alpha_{1}+\alpha_{2}}{2} \\
d_{1}=d_{3}=d_{2}=d_{4}=2 r
\end{array}\right.
$$

The dimensionless total surface energy in a meso-structure $E_{s}^{m e s o^{*}}$ can therefore be written as:

$$
E_{s}^{m e s o^{*}}=8 E_{s}^{b r i d g e^{*}}\left(V_{i}^{*}\right)+4 E_{s}^{b r i d g e^{*}}\left(V_{v}^{*}\right)=8 E_{s}^{b r i d g e^{*}}\left(\frac{V_{w}^{*}-4 V_{v}^{*}}{8}\right)+4 E_{s}^{b r i d g e^{*}}\left(V_{v}^{*}\right)
$$

The derivative of the total surface energy with respect to the volume of one vertical bridge is:

$$
\frac{\mathrm{d} E_{s}^{\text {meso* }}}{\mathrm{d} V_{v}^{*}}=-4 \frac{\mathrm{d} E_{s}^{\text {bridge }}{ }^{*}}{\mathrm{~d} V_{v}^{*}}\left(\frac{V_{w}^{*}-V_{v}^{*}}{4}\right)+4 \frac{\mathrm{d} E_{s}^{\text {bridge }}}{\mathrm{d} V_{v}^{*}}\left(V_{v}^{*}\right)
$$

Figure 11 shows the surface energy in a bridge. The minimum of $E_{s}^{m e s o^{*}}$, given by $\frac{d E_{s}^{m e s o^{*}}}{d V_{v}^{*}}=0$ corresponds to the case where $V_{i}^{*}=V_{v}^{*}=\frac{V_{w}^{*}}{12}$.

To insure that the bridges do not touch each other, the filling angles of the vertical bridges $\beta_{v}$ and of the inclined bridges $\beta_{i}$, as defined in Figure 12, should fulfil the following conditions:

$$
\left\{\begin{array}{l}
\cos \beta_{i}>\cos \beta_{i, \text { coal }}=\sqrt{\frac{1+\cos ^{2} \alpha}{2}} \\
\beta_{v}>\beta_{v, \text { coal }}=\pi-\alpha-\beta_{i}
\end{array}\right.
$$

where $\beta_{i, \text { coal }}$ is the limit half-filling angle of the inclined bridges corresponding to the contact of the inclined bridge and $\beta_{v \text {,coal }}$ is the limit half-filling angle of the vertical bridges corresponding to the contact between inclined and vertical bridges. 


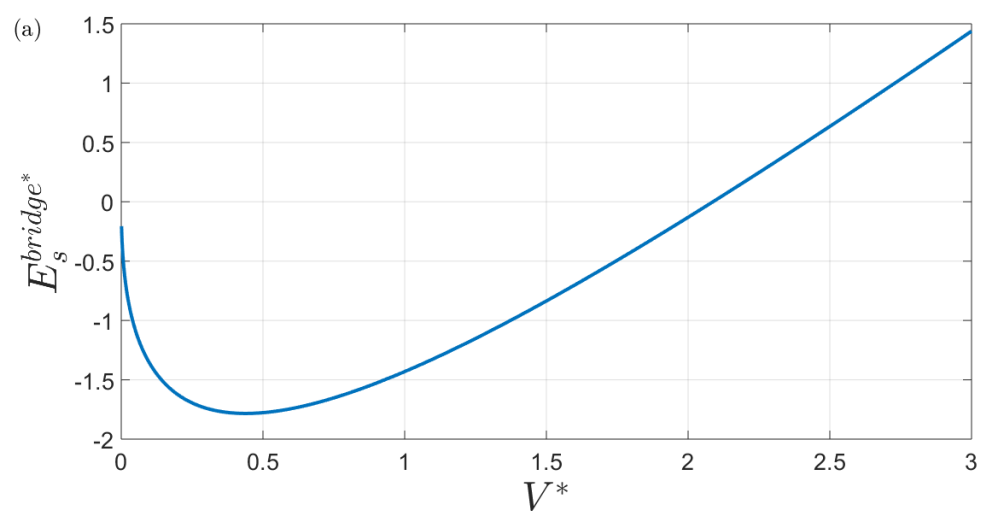

Fig. 11: Surface energy in a liquid bridge between two grains in contact, as a function of the water volume.

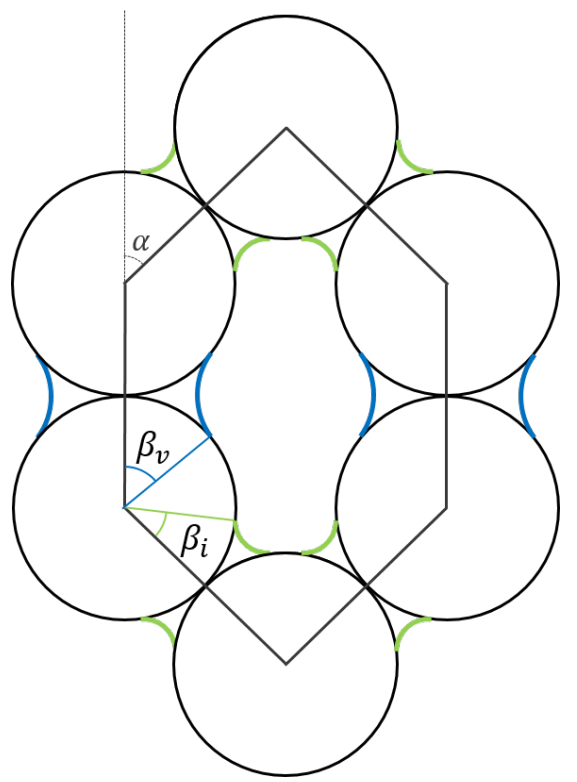

Fig. 12: Definition of the vertical filling angle $\beta_{v}$ and the inclinated filling angle $\beta_{i}$.

The conditions of contact defined by Equation 33 are checked for $V_{i}^{*}=V_{w}^{*} / 12$. In case of contact, $V_{i}$ is decreased to the maximal value which insure the independence of the capillary bridges, in order to obtain the geometrically compatible configuration with the minimal surface energy.

At this point, volume in each bridge is known, and the capillary force at each contact of the cell can be deduced from the Equation 27. These capillary forces, noted $N_{1}^{c a p}$ or $N_{3}^{c a p}$ in the inclined bridges and $N_{2}^{c a p}$ or $N_{4}^{c a p}$ in the vertical bridges are added to the contact forces. At mesoscale, $\widetilde{V} \widetilde{\boldsymbol{\sigma}}$ are replaced by $\widetilde{V} \widetilde{\boldsymbol{\sigma}}^{\text {cap }}$ in Equation 20 and Equation 17, thus, using Love-Weber formula:

$$
\begin{aligned}
\widetilde{V} \widetilde{\sigma}_{11}^{c a p}= & 4\left(N_{1}+N_{1}^{c a p}\right) d_{1} \cos ^{2} \alpha_{1}+4 T_{1} d_{1} \cos \alpha_{1} \sin \alpha_{1}+2\left(N_{2}+N_{2}^{c a p}\right) d_{2} \\
& +4\left(N_{3}+N_{3}^{c a p}\right) d_{3} \cos ^{2} \alpha_{2}+4 T_{3} d_{3} \cos \alpha_{2} \sin \alpha_{2}+2\left(N_{4}+N_{4}^{c a p}\right) d_{4} \\
\widetilde{V} \widetilde{\sigma}_{22}^{c a p}= & 4\left(N_{1}+N_{1}^{c a p}\right) d_{1} \sin ^{2} \alpha_{1}-4 T_{1} d_{1} \cos \alpha_{1} \sin \alpha_{1} \\
\widetilde{V} \widetilde{\sigma}_{33}^{c a p}= & 4\left(N_{3}+N_{3}^{c a p}\right) d_{3} \sin ^{2} \alpha_{2}-4 T_{3} d_{3} \cos \alpha_{2} \sin \alpha_{2} \\
& \boldsymbol{\sigma}=\frac{1}{V} \iiint \omega(\theta, \varphi, \psi) \boldsymbol{P}^{-1} \widetilde{V} \tilde{\boldsymbol{\sigma}}^{c a p}(\boldsymbol{n}, \boldsymbol{t}, \boldsymbol{w}) \boldsymbol{P} \sin \varphi d \varphi d \theta d \psi
\end{aligned}
$$

When considering a large deformation problem such as sand column collapse, an invariant stress tensor with respect to rigid-body rotation must be applied. Accordingly, the Green-Naghdi rate which gives an objective measure of the stress rate was adopted in this study (Green and Naghdi, 1964; Fávero Neto and Borja, 2018; Fávero Neto et al., 2020; Liang and Zhao, 2019; Jin et al., 2020; Systèmes, 2014):

$$
\boldsymbol{\sigma}^{\nabla G}=\dot{\boldsymbol{\sigma}}-\boldsymbol{\Omega} \cdot \boldsymbol{\sigma}+\boldsymbol{\sigma} \cdot \boldsymbol{\Omega}^{T}
$$


Table 1: Parameters selected in calibration and prediction phases

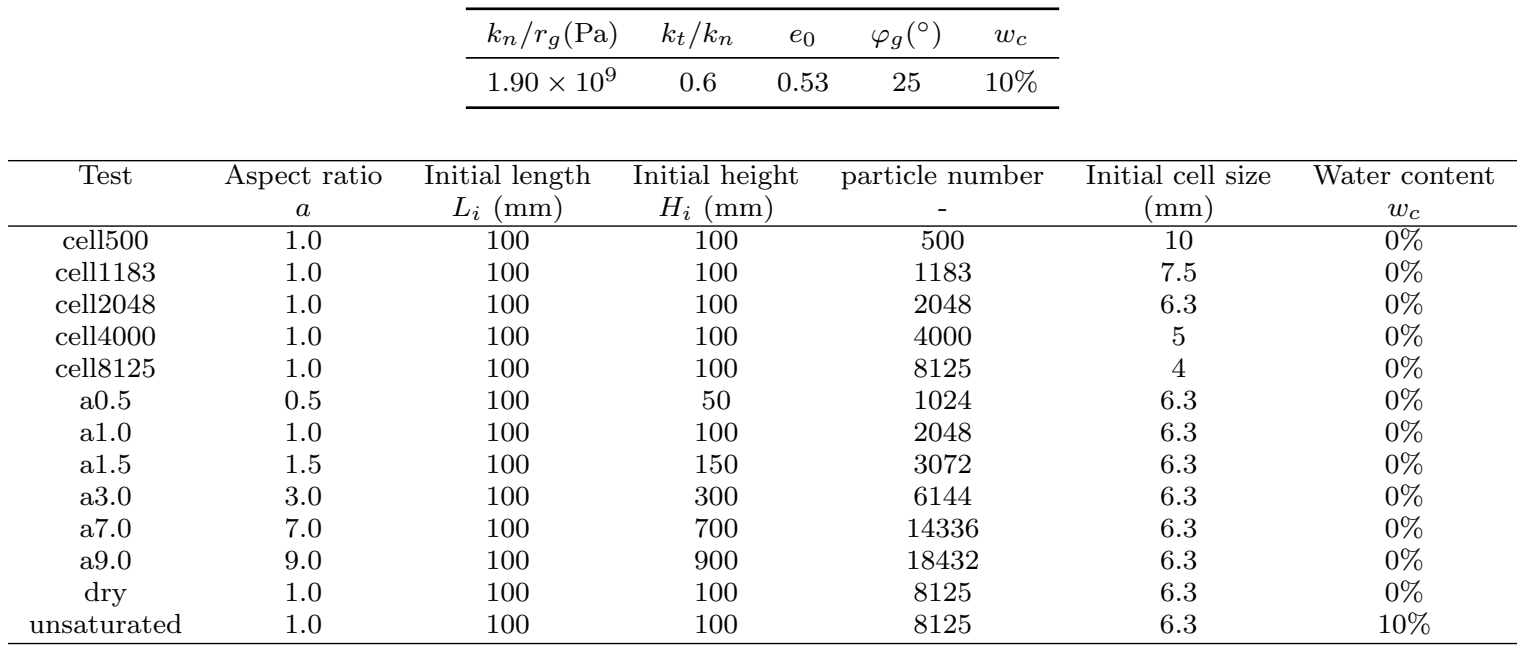

Table 2: Dimension and discretization parameters for all numerical simulations

where $\boldsymbol{\Omega}=\dot{\boldsymbol{R}} \cdot \boldsymbol{R}^{T}, \boldsymbol{R}$ is the rigid body rotation in the polar decomposition of the deformation gradient $\boldsymbol{F}$. The differences between Green-Naghdi rate and Jaumann rate are significant only if finite rotation of a material point is accompanied by finite shear.

\subsection{Implementation of 3D-H model in SPH}

The implementation of the 3D-H model in the SPH codes constitutes a complete multi-scale procedure. The SPH method is an effective method to solve the large deformation boundary value problem. SPH searches whether particles $\mathrm{i}$ and $\mathrm{j}$ interact with each other. Once determined that the two particles interact, the statistical representative volume element (RVE) of the 3D-H model embedded in the SPH particles begins to work. As illustrated before, the 3D-H model localizes the incremental macro strain tensor by using Equation 7. Then, the meso-structures are decomposed into two perpendicular hexagons, in which the constitutive behaviour is able to be solved (Equation 13). Hereafter, the incremental macro stress tensor of the RVE is obtained by stress averaging (Equation 17). Finally, the position and velocity of SPH particles are updated. The entire calculation process of the proposed multi-scale approach is shown in Figure 13.

\section{Multi-scale modelling of granular collapse}

\subsection{Model setup}

The numerical example chosen for the demonstration of the proposed multi-scale approach is a classical dynamic problem: the two-dimensional granular column collapsed to frictional ground under gravity. The geometry and the boundary conditions of the granular column are shown in Figure 14. $L_{i}$ and $H_{i}$ denote the initial length and initial height, respectively. The initial aspect ratio is defined as $a=H_{i} / L_{i}$. After granular columns collapse to finial deposit profile, $L_{f}$ and $H_{f}$ denote the run-out distance and deposit height, respectively. Except for the ground, other boundary walls are frictionless walls. The contact between SPH particles and the frictional wall is described by the classical Coulomb friction law with a friction coefficient of 0.3. Dimension and discretization parameters for all numerical simulations are summarized in Table 2. The parameters used in the extended 3D-H model is reported in Table 1. The process of numerical simulation is divided into two stages: (1) SPH particles are generated and are reached the self-weight balance; (2) move up the right wall with a speed of $1 \mathrm{~m} / \mathrm{s}$. It is worth noting that the initial stress of SPH particles is set to balance with the self-weight. Due to the particularity of the micro-mechanical model, all RVEs need to be confined up to the initial stress to obtain the state variables at the microscopic scale, just like DEM. During the calculation, six representative SPH particles (A-F marked by red cross in Figure 14a) are selected for meso-scale analysis. 


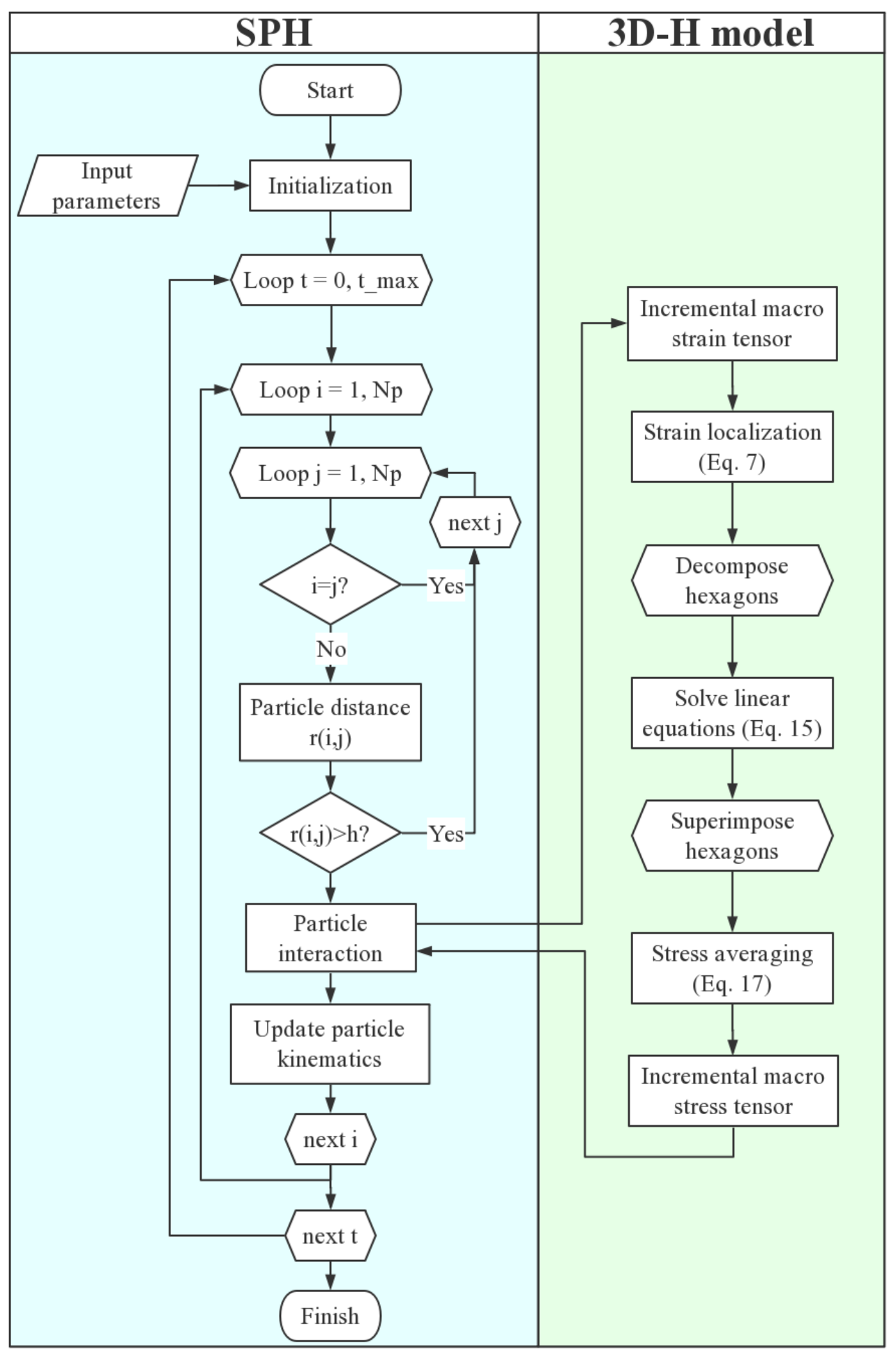

Fig. 13: Flowchart of the proposed multi-scale approach. 


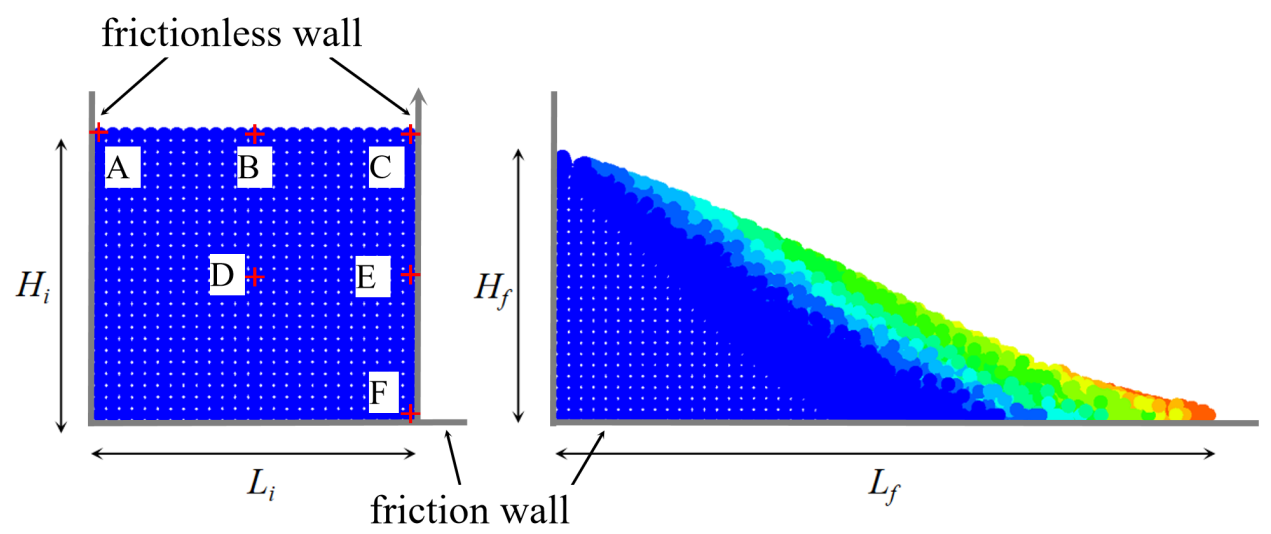

(a)

(b)

Fig. 14: Model setup and final deposit configurations for the collapse of granular column: (a) initial sample; (b) final deposit $\left(L_{i}\right.$ : initial length; $H_{i}$ : initial height; $L_{f}$ : run-out distance; $H_{f}$ : deposit height). Selected particles for meso-scale analysis is marked as red cross

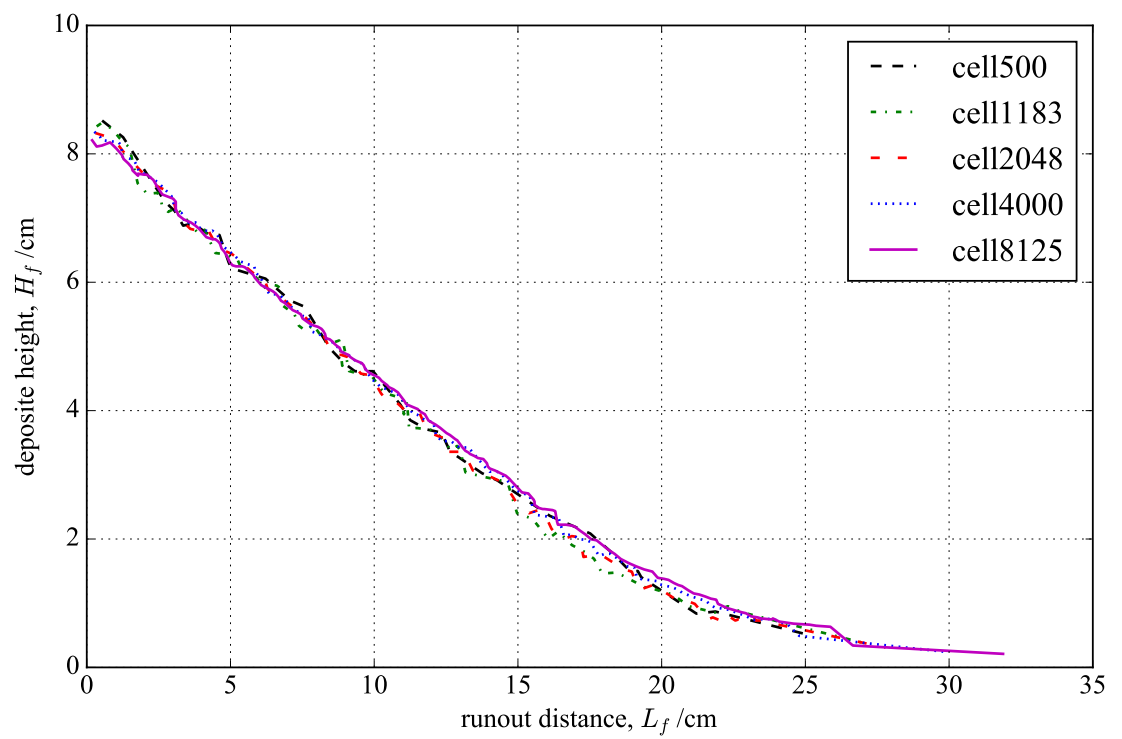

Fig. 15: Comparison of final deposition profiles between different initial cell sizes.

\subsection{Effect of initial cell size}

Negligence of initial cell size can sometimes be an embarrassment in SPH, especially in large deformation problems and dynamic problems. Thus the effect of initial cell size of the proposed multi-scale approach is first examined. Five tests with different numbers of SPH particles are performed. Figure 15 shows a comparison of the final deposition profile between different initial cell size tests. The results show that there are slight differences that are negligible.

\subsection{Performance of the proposed approach}

The problem of granular column collapse has been extensively studied in the literature. For granular flows, the assessment of the final run-out distance is crucially significant, as it determines the extent of the regions affected by the avalanches or landslides. In this section, our simulations have been compared with previous numerical results from (Staron and Hinch, 2005; Crosta et al., 2009; Utili et al., 2015) and experimental observations from (Lajeunesse et al., 2005; Lube et al., 2005) in the literature. Following the dimensional analysis by (Utili et al., 2015), the final normalized deposit height is defined as $[H]=H_{f} / L_{i}$ while the final normalized run-out distance is defined as $[L]=\left(L_{f}-L_{i}\right) / L_{i}$. Figure 16 shows the final normalized deposit height $[H]$ and the final normalized run-out distance $[L]$ versus different initial aspect ratio. As 


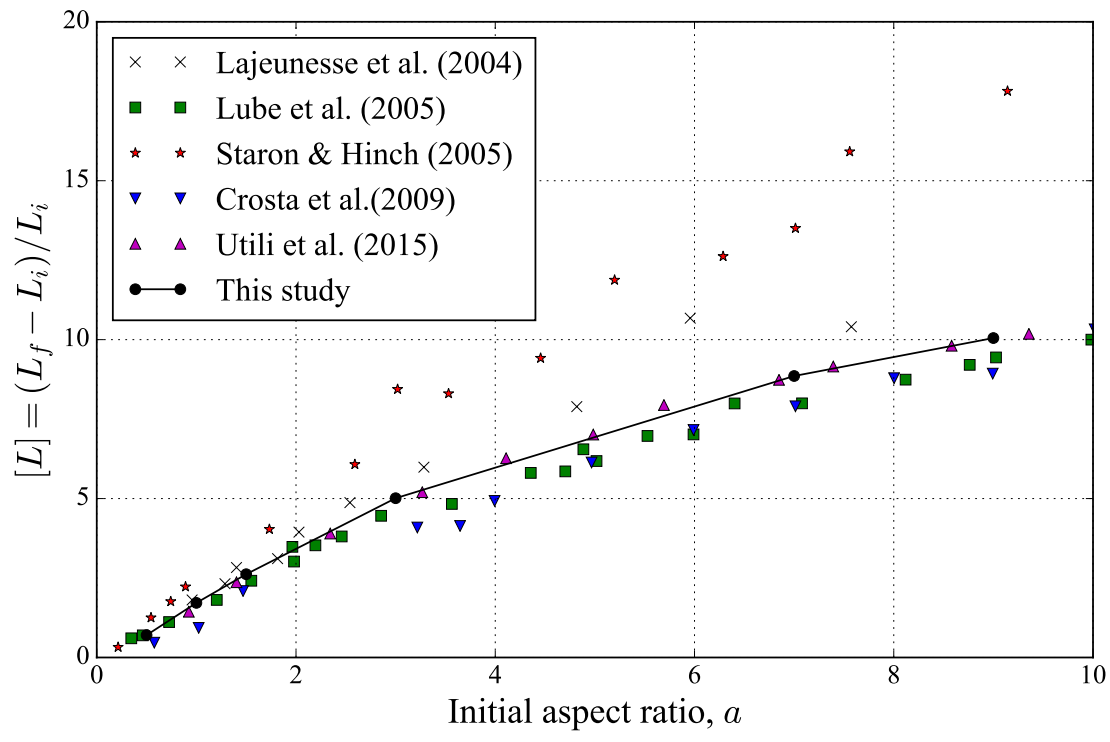

(a) Final normalized run-out distance

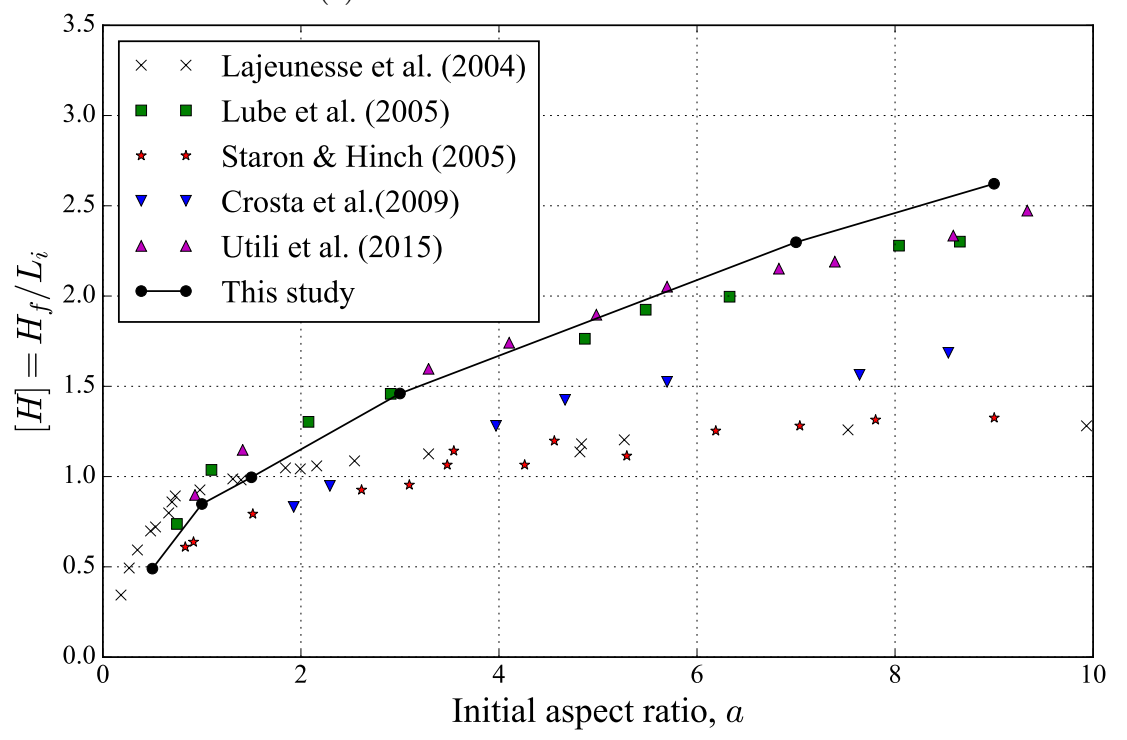

(b) Final normalized deposit height

Fig. 16: Comparison between the numerical results $\left(w_{c}=0 \%\right)$ of this study and results from literatures.

shown in Figure 16a, it can be observed that the final normalized run-out distance predicted by this study shows good agreement with experimental observations and numerical results from a qualitative viewpoint, including (Lube et al., 2005) from experimental tests performed under plane strain conditions, the 3D DEM numerical results from (Utili et al., 2015), and the 2D FEM numerical results from (Crosta et al., 2009). The difference between experimental observations from (Lajeunesse et al., 2005) and the results obtained by this study probably stems from the usage of glass beans. Since particle angularity tends to reduce run-out, the unrealistically large run-out distance is observed by (Lajeunesse et al., 2005). Similarly, unrealistically large run-out distance is also observed by (Staron and Hinch, 2005), due to the fact that 2D DEM simulations are employed. Meanwhile, the final normalized deposit height shown in Figure 16b has similar observations as the final normalized run-out distance. The predicted results are consistent with the results obtained by (Lube et al., 2005; Utili et al., 2015; Crosta et al., 2009), but lower than (Lajeunesse et al., 2005; Staron and Hinch, 2005). It can be concluded that the proposed approach is able to predict the final deposit morphology of collapse of granular column.

The prediction of the final deposit profile by the proposed approach was discussed previously, below we will focus on the collapse process. In Figure 17, the evolutions of normalized granular spreading length versus normalized time is plotted for various initial aspect ratio. Following (Lube et al., 2005; Crosta et al., 2009), time has been normalized by $[t]=t / \sqrt{H_{i} / g}$, which can be thought of as the time taken by a single particle in free fall to travel from the centre of the column to the base. As shown in the figure, the 


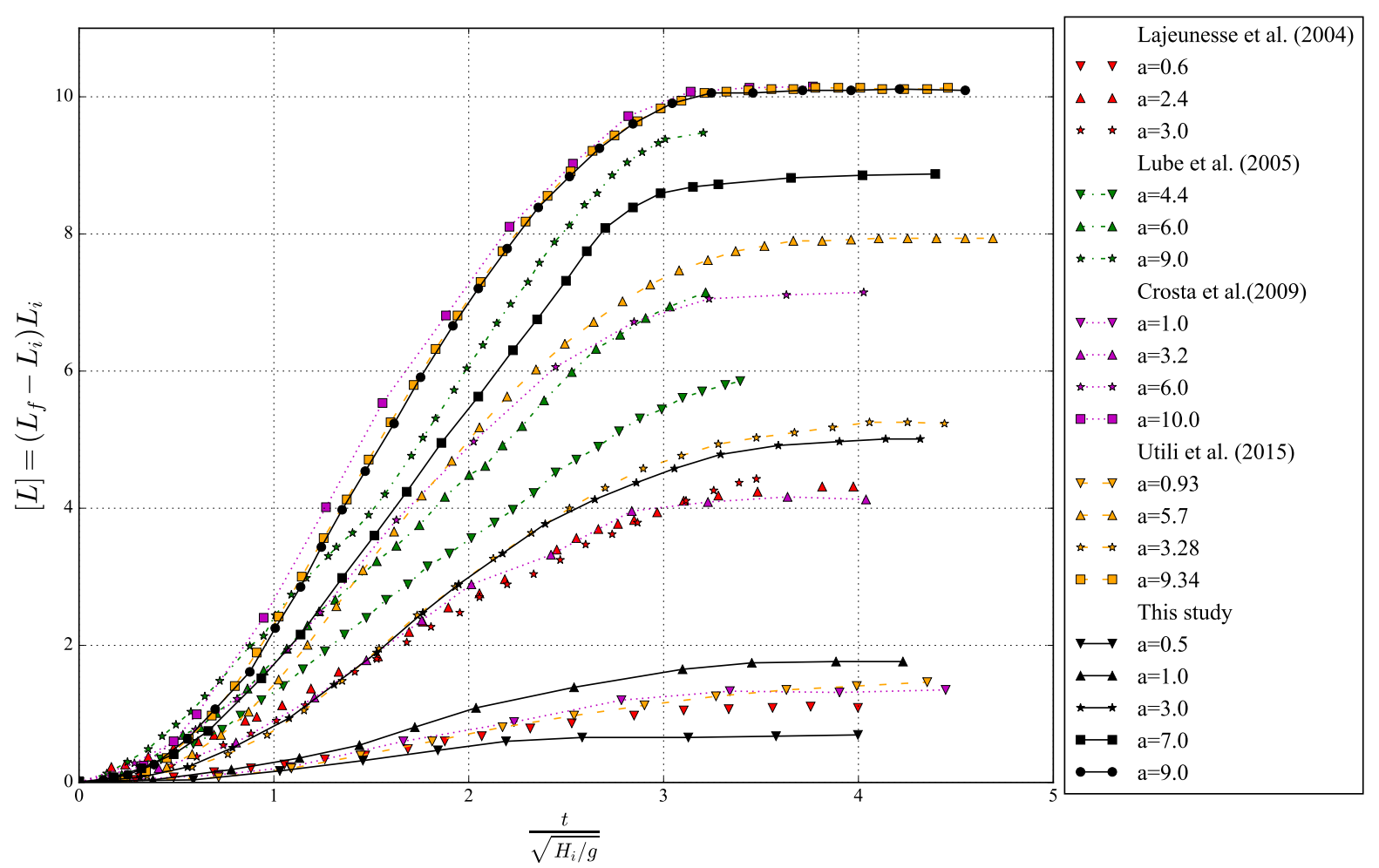

Fig. 17: Normalized granular spreading length versus normalized time of experimental and numerical $\left(w_{c}=\right.$ $0 \%$ ) results.

development of the curve of all the results can be roughly divided into four stages: $(1) 0 \leqslant[t]<1$, the onset of collapse with an initial transient acceleration; (2) $1 \leqslant[t]<2$, the development of collapse with a constant velocity flow; (3) $2 \leqslant[t]<3$ stabilizing of granular flow with a gradual deceleration; (4) $[t] \geq 3$ final deposit of granular flow. By comparing the numerical results obtained by this study with the results from literatures, it can be found that the predicted results by the proposed approach located in a reasonable range. This verifies the correctness of the proposed multi-scale approach from another point of view.

Certainly, the verification of Figure 17 is only from the perspective of the spreading length of granular column collapse. For the sake of concision, the test $a=1.0$ is selected for a detailed comparison of the collapse process. Figure 18 shows the comparison of failure progress of granular collapse between numerical results obtained by proposed approach and experimental observations from (Nguyen et al., 2017) for initial aspect ratio $\mathrm{a}=1.0$ for both dry and unsaturated materials. The results of dry material are shown on the left column while the unsaturated case with water content $w_{c}=10 \%$ are shown on the right column. It is worth noting that the velocity fields obtained by numerical calculations are all extracted in strict accordance with the time points recorded in the experiment. In general, the simulated predictions are basically consistent with the observed morphological evolution. Since the experimental results are based on dry conditions, the numerical results of dry case is closer to the experimental results. It also implies that the proposed approach can not only predict the final deposit state, but also capture the whole process of granular column collapse problem. The comparison between the dry and unsaturated conditions shows that the capillary forces delay the collapse and reduces the run-out distance of the granular column. This observation is qualitatively in agreement with experimental results obtained by (Gabrieli et al., 2013) and reproduced numerically by (Wang et al., 2019). Quantitative comparison are out of the scope of the present paper but will be performed in forthcoming works.

\subsection{Meso-scale analysis}

Taking advantage of the 3D-H model, a meso-scale analysis can be performed. The key microscopic behaviours hiding behind the macroscopic scale are helpful to understand and interpret the micro-mechanisms governing the overall response. Six representative SPH particles (A-F, marked as red cross in Figure 14a) are selected. Figure 19 and Figure 20 reveal the microscopic variable distributions of 3D-H model inside SPH particles at three states of dry case: (1) initial state at $\mathrm{t}=0 ;(2)$ developing of collapse at $\mathrm{t}=0.118$ 

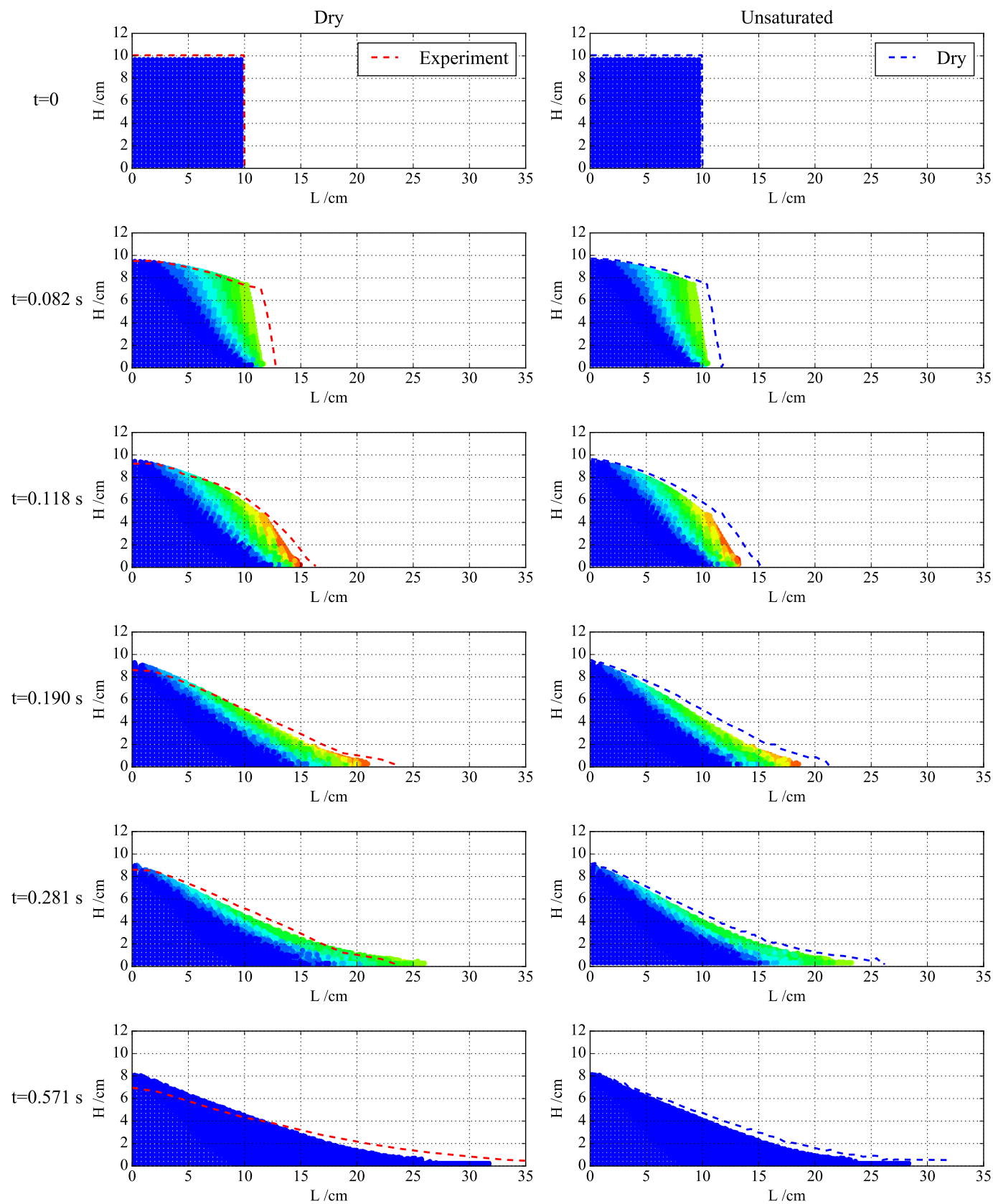

Fig. 18: Comparison of failure progress of granular collapse between simulations and experiments for initial aspect ratio $(a=1)$ : left column shows the results of dry material while right column shows the results of unsaturated material with water content $w_{c}=10 \%$.

$\mathrm{s}$; and (3) final deposit state at $\mathrm{t}=0.571 \mathrm{~s}$. For the sake of simplification, the original 3D distributions of hexagons are plotted in 2D by integrating over two Euler angles $\theta$ and $\psi$ as follows:

$$
\begin{aligned}
& \tilde{\sigma}_{n}^{I}(\varphi)=\iint \omega \widetilde{\sigma}_{n} d \theta d \psi \\
& \widetilde{\sigma}_{t}^{I}(\varphi)=\iint \omega \widetilde{\sigma}_{t} d \theta d \psi
\end{aligned}
$$

where $\widetilde{\sigma_{n}}$ and $\widetilde{\sigma_{t}}$ are normal and tangential stress on the meso-scale, thus $\widetilde{\sigma}_{n}^{I}(\varphi)$ and $\widetilde{\sigma}_{t}^{I}(\varphi)$ can be considered as a function of the last Euler angle $\varphi$, it should be noted that $\varphi=0^{\circ}$ coincides with the positive z-axis direction in the global coordinate system.

The first observation is that all mesoscopic variables are isotropically distributed when $\mathrm{t}=0 \mathrm{~s}$, as mentioned earlier. The initial magnitude of the mesoscopic variables at each selected point is different, depending on the initial stress at which the point is located. As the collapse progressed, the distribution of $\widetilde{\sigma}_{n}^{I}(\varphi)$ and $\tilde{\sigma}_{t}^{I}(\varphi)$ gradually became anisotropic, indicating that force fabric gradually evolved into anisotropy. This is a natural evolution of the 3D-H model, with no parameter control. In response to the large deformation 


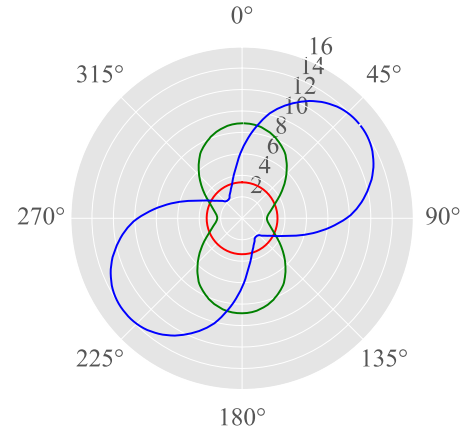

$\tilde{\sigma}_{n}^{I}(\varphi)(\mathrm{Pa})$

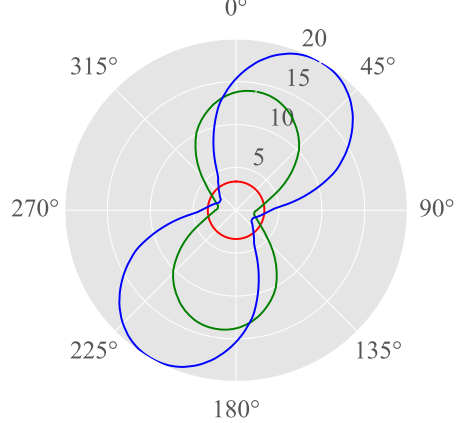

$\tilde{\sigma}_{n}^{I}(\varphi)(\mathrm{Pa})$

$0^{\circ}$

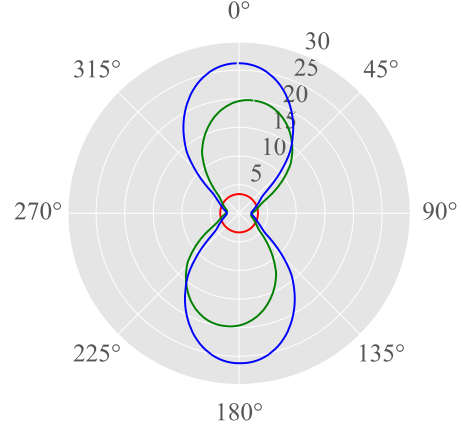

$\tilde{\sigma}_{n}^{I}(\varphi)(\mathrm{Pa})$

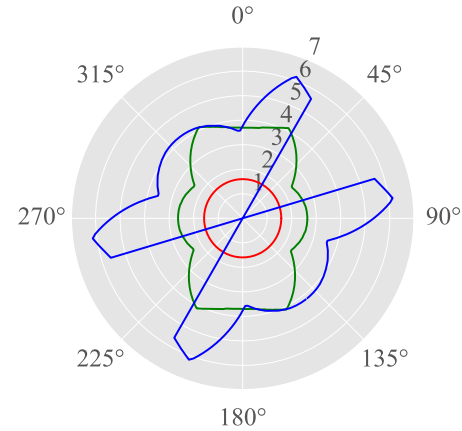

$\tilde{\sigma}_{t}^{I}(\varphi)(\mathrm{Pa})$

(a) Point $\mathrm{A}$

$0^{\circ}$

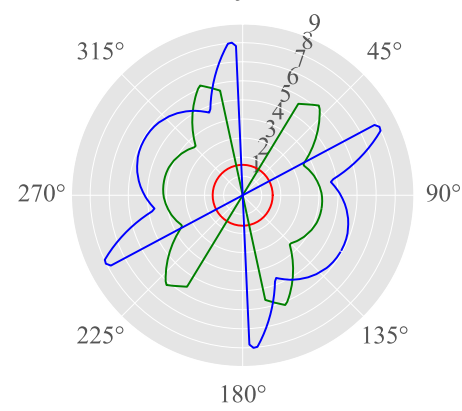

$\tilde{\sigma}_{t}^{I}(\varphi)(\mathrm{Pa})$

(b) Point B

$0^{\circ}$

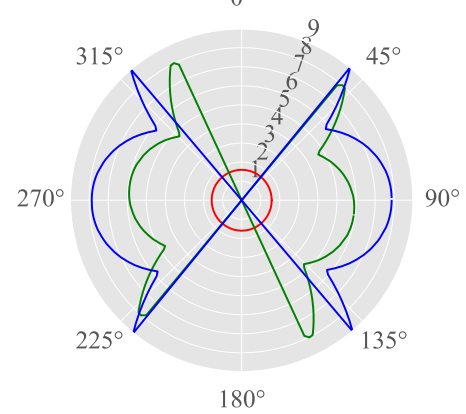

$\tilde{\sigma}_{t}^{I}(\varphi)(\mathrm{Pa})$

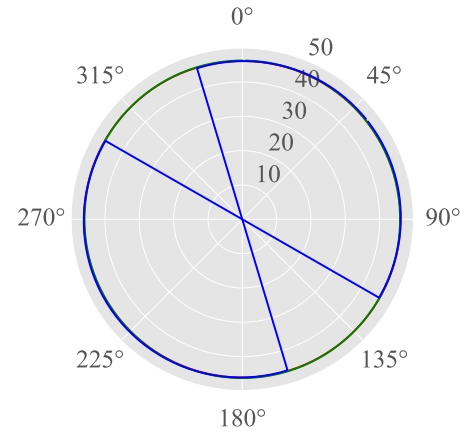

$\alpha_{2}\left({ }^{\circ}\right)$

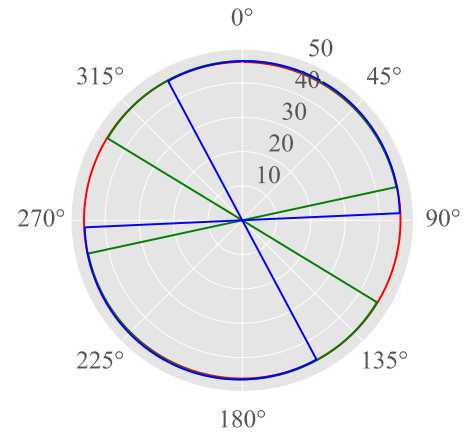

$\alpha_{2}\left({ }^{\circ}\right)$

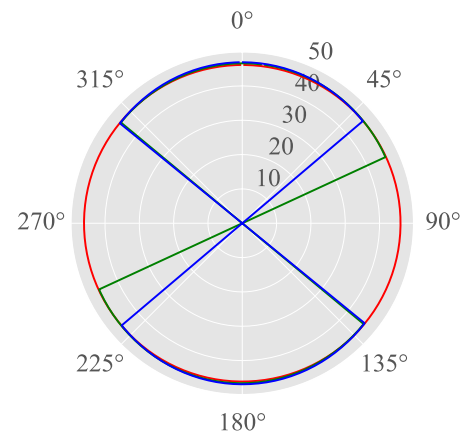

$\alpha_{2}\left({ }^{\circ}\right)$

(c) Point C

$-\mathrm{t}=0.000 \mathrm{~s}-\mathrm{t}=0.118 \mathrm{~s}-\mathrm{t}=0.571 \mathrm{~s}$

Fig. 19: Meso-scale variable distributions of 3D-H model inside SPH particles A, B and C at three states. The selected SPH particle positions are marked as red cross in Figure 14a.

dynamics problem, such as granular column collapse, the proposed approach is dedicated to simulating macroscopic stress-strain response based on the evolution of microscopic and mesoscopic information. Naturally, In granular materials, the change in contact fabric is due to the disconnection of existing contacts and the re-contact of new contacts. In the current version of the 3D-H model, when a meso-structure fails, it is not lost, but stored in the system with a 'no-contribution' label. If the global deformation develops and makes this opening contact re-contact, the failed meso-structure is reactivated. However, we do mention that in the current version of the model, no new local meso-structure can appear during a loading program. The meso-scale analysis helps to compare the observations by micro-CT or results by DEM simulations and further improve the model, especially in an actual boundary value problem. 


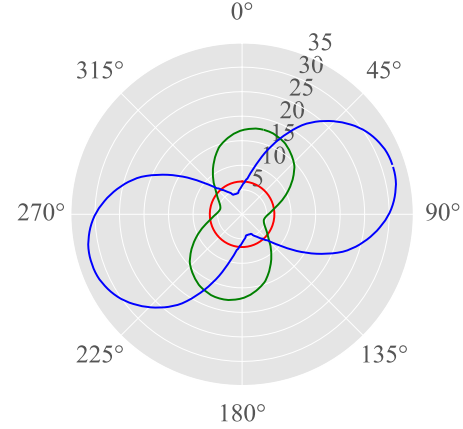

$\tilde{\sigma}_{n}^{I}(\varphi)(\mathrm{Pa})$

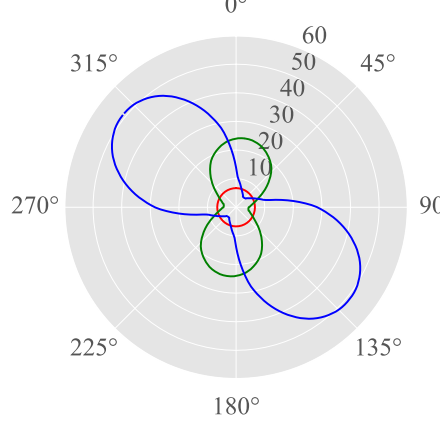

$\tilde{\sigma}_{n}^{I}(\varphi)(\mathrm{Pa})$

$0^{\circ}$

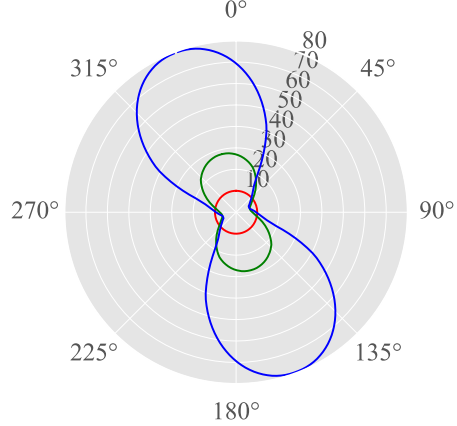

$\tilde{\sigma}_{n}^{I}(\varphi)(\mathrm{Pa})$

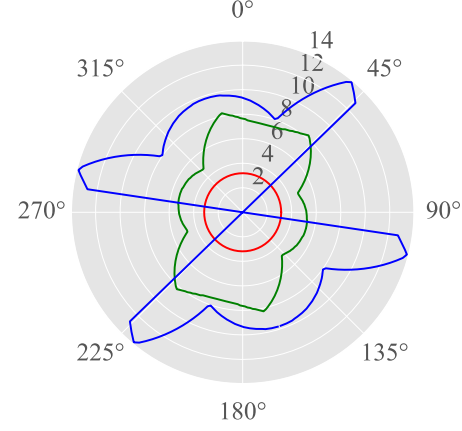

$\tilde{\sigma}_{t}^{I}(\varphi)(\mathrm{Pa})$

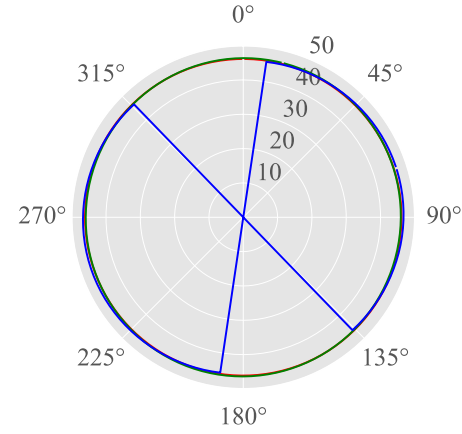

$\alpha_{2}\left({ }^{\circ}\right)$

(a) Point D

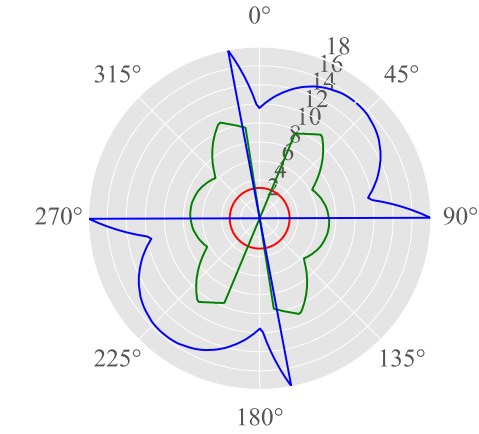

$\tilde{\sigma}_{t}^{I}(\varphi)(\mathrm{Pa})$

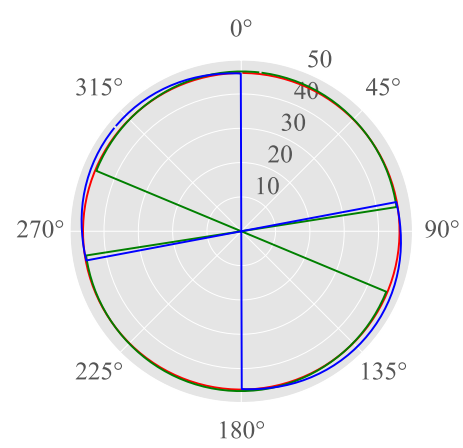

$\alpha_{2}\left({ }^{\circ}\right)$

(b) Point E

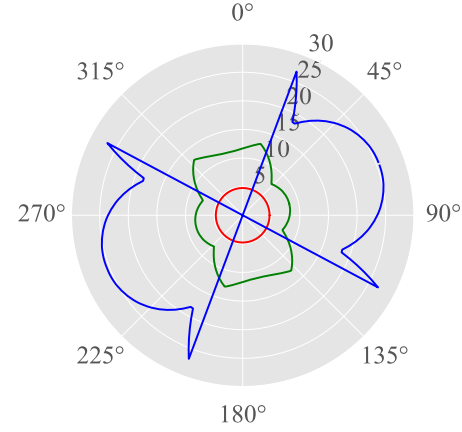

$\tilde{\sigma}_{t}^{I}(\varphi)(\mathrm{Pa})$

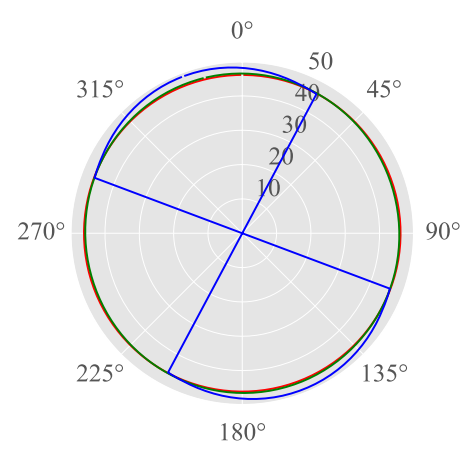

$\alpha_{2}\left({ }^{\circ}\right)$

(c) Point F

Fig. 20: Meso-scale variable distributions of 3D-H model inside SPH particles D, E and F at three states. The selected SPH particle positions are marked as red cross in Figure 14a. 


\section{Conclusions}

This paper presents a new multi-scale coupling approach for simulation of granular column collapse without using the traditional phenomenological constitutive models. The SPH method is employed to solve large deformation boundary value problems while the 3D-H model is used to derive the necessary constitutive response for the representative volume element embedded in each material point of the SPH. A preliminary attempt of the 3D-H model for a low water content condition has been made by taking into account the capillary force between connected grains. The main results are summarized as below:

1. The impact of capillary forces for small water contents is noticeable and impacts both the dynamic of the collapse and the run-out distance of the granular column. Nevertheless, the impact for small water contents is shown to be quantitatively limited. The maximum water content that can be presently reached is limited by the pendular regime approximation. Capillary forces calculation in 3D-H model for higher water contents, for which pendular bridges merge, is currently being investigated.

2. With regard to the large deformation problem, the multi-scale approach can not only capture the final deposition profiles of the granular material after large deformation, but also capture the microstructure failure process from small deformation to large deformation.

3. The final deposit profiles of granular column predicted by the proposed approach quantitatively match the DEM analysis by (Utili et al., 2015) and experimental results by (Lube et al., 2005). The evolution of final normalized run-out distance $[L]$ and deposit height $[H]$ versus initial aspect ratio $\alpha$ are consistent with the results by (Lajeunesse et al., 2005; Staron and Hinch, 2005; Crosta et al., 2009).

4. Different four stages have been observed in the evolution of normalized run-out distance $[L]$ versus normalized time $[t]$. The whole process of collapse is consistent with the experimental observations by (Nguyen et al., 2017).

5. Owing to the advantages of the proposed multi-scale approach, the meso-scale analysis is performed within six material points in SPH. It shows how the mesoscopic variables evolve during such a dynamic large deformation problem. It also helps to the further improvements of the micromechanical model by comparing with the observations by micro-CT or numerical results by DEM.

Finally, the coupling multi-scale approach provides an effective computational tool for the analysis of granular flows.

\section{Acknowledgment}

The financial support provided by the GRF project (Grant No. 15209119) and the RIF project (Grant No. PolyU R5037-18F) from the Research Grants Council (RGC) of Hong Kong are gratefully acknowledged. We also gratefully acknowledge the CNRS International Research Network GeoMech for having offered the opportunity to make this project possible through a long term collaboration of all the authors (http://gdrmege.univ-lr.fr/).

\section{A Contact law}

This elastic-perfect plastic model includes a Mohr-Coulomb criterion and can be expressed under the following incremental formalism:

$$
\left\{\begin{array}{l}
\delta N_{i}=k_{n} \delta u_{n}^{i} \\
\delta T_{i}=\min \left\{\left\|T_{i}+k_{t} \delta u_{t}^{i}\right\|, \tan \varphi_{g}\left(N_{i}+k_{n} \delta u_{n}^{i}\right)\right\} \times \frac{T_{i}+k_{t} \delta u_{t}^{i}}{\left\|T_{i}+k_{t} \delta u_{t}^{i}\right\|}-T_{i}
\end{array}\right.
$$

where: $i=1,2,3,4$ denotes the identifier of contact number.

According to Equations 8, Equations 38 can be rewritten as follows:

$$
\left\{\begin{array}{lr}
\delta N_{i}=-k_{n} \delta d_{i} & \\
\delta T_{i}=k_{t} d_{i} \delta \alpha_{j} & \text { elastic regime } \\
\delta T_{i}=\tan \varphi_{g}\left(N_{i}-k_{n} \delta d_{i}\right) \xi_{i}-T_{i} & \text { plastic regime }
\end{array}\right.
$$

where: $\xi_{i}$ is the $\operatorname{sign}$ of $T_{i}+k_{t} d_{i} \delta \alpha_{j} ; j=1$ when $i=1,2 ; j=2$ when $i=3,4$; plastic regime is reached when $\left\|k_{t} d_{i} \delta \alpha_{j}+T_{i}\right\| \geqslant \tan \varphi_{g}\left(N_{i}-k_{n} \delta d_{i}\right)$, otherwise it is in elastic regime.

To facilitate the derivation, $I_{i}^{p}$ and $I_{i}^{e}$ are introduced as indicator functions of the contact state, expressed as follow:

$$
I_{i}^{p}=\left\{\begin{array}{ll}
1 & \text { in plastic regime } \\
0 & \text { in elastic regime }
\end{array} ; \quad I_{i}^{e}=1-I_{i}^{p}\right.
$$

Thus, the constitutive relations can be expressed as:

$$
\left\{\begin{array}{l}
\delta N_{i}=-k_{n} \delta d_{i} \\
\delta T_{i}=B_{i} \delta \alpha_{j}-A_{i} \delta d_{i}+C_{i}
\end{array}\right.
$$


where: $\left\{\begin{array}{l}A_{i}=I_{i}^{p} k_{n} \xi_{i} \tan \varphi_{g} \\ B_{i}=I_{i}^{e} k_{t} d_{i} \\ C_{i}=I_{i}^{p}\left(\xi_{i} \tan \varphi_{g} N_{i}-T_{i}\right)\end{array}\right.$ 


\section{References}

Brakke, K.A., 1992. The surface evolver. Experimental Mathematics 1, 141-165.

Brezzi, L., Gabrieli, F., Cola, S., 2020. Collapse of granular-cohesive soil mixtures on a horizontal plane. Acta Geotechnica $15,695-714$

Bui, H.H., Fukagawa, R., Sako, K., Ohno, S., 2008. Lagrangian meshfree particles method (sph) for large deformation and failure flows of geomaterial using elastic-plastic soil constitutive model. International journal for numerical and analytical methods in geomechanics 32, 1537-1570.

Cambou, B., Dubujet, P., Emeriault, F., Sidoroff, F., 1995. Homogenization for granular materials. European journal of mechanics. A. Solids 14, 255-276.

Chen, W.B., Liu, K., Feng, W.Q., Borana, L., Yin, J.H., 2019. Influence of matric suction on nonlinear time-dependent compression behavior of a granular fill material. Acta Geotechnica , 1-19.

Chen, W.B., Liu, K., Yin, Z.Y., Yin, J.H., 2020. Crushing and flooding effects on one-dimensional time-dependent behaviors of a granular soil. International Journal of Geomechanics 20, 04019156.

Christoffersen, J., Mehrabadi, M.M., Nemat-Nasser, S., 1981. A micromechanical description of granular material behavior. Journal of Applied Mechanics 48, 339-344.

Crosta, G., Imposimato, S., Roddeman, D., 2009. Numerical modeling of 2-d granular step collapse on erodible and nonerodible surface. Journal of Geophysical Research: Earth Surface 114.

De Saxcé, G., Fortin, J., Millet, O., 2004. About the numerical simulation of the dynamics of granular media and the definition of the mean stress tensor. Mechanics of Materials 36, 1175-1184.

Fávero Neto, A.H., Askarinejad, A., Springman, S.M., Borja, R.I., 2020. Simulation of debris flow on an instrumented test slope using an updated Lagrangian continuum particle method. Acta Geotechnica 1. doi:10.1007/s11440-020-00957-1.

Fávero Neto, A.H., Borja, R.I., 2018. Continuum hydrodynamics of dry granular flows employing multiplicative elastoplasticity. Acta Geotechnica 13, 1027-1040. doi:10.1007/s11440-018-0700-3.

Fern, E.J., Soga, K., 2017. Granular column collapse of wet sand. Procedia Engineering 175, 14-20.

Gabrieli, F., Artoni, R., Santomaso, A., Cola, S., 2013. Discrete particle simulations and experiments on the collapse of wet granular columns. Physics of fluids 25, 103303.

Gingold, R.A., Monaghan, J.J., 1977. Smoothed particle hydrodynamics: theory and application to non-spherical stars. Monthly notices of the royal astronomical society 181, 375-389.

Green, A.E., Naghdi, P.M., 1964. A general theory of an elastic-plastic continuum. Technical Report. CALIFORNIA UNIV BERKELEY INST OF ENGINEERING RESEARCH.

Han, K., Ju, J.W.W., Kong, H., Wang, M., 2019. Functional Catastrophe Analysis of Progressive Failures for Deep Tunnel Roof Considering Variable Dilatancy Angle and Detaching Velocity. Rock Mechanics and Rock Engineering 52, 39873997.

Hungr, O., Evans, S., 2004. Entrainment of debris in rock avalanches: an analysis of a long run-out mechanism. Geological Society of America Bulletin 116, 1240-1252.

Iverson, R.M., 1997. The physics of debris flows. Reviews of geophysics 35, 245-296.

Jianbo, F., Yuxin, J., Xiaohui, S., Xi, C., 2020. Experimental investigation on granular flow past baffle piles and numerical simulation using a $\mu(\mathrm{I})$-rheology-based approach. Powder Technology 359, 36-46. doi:10.1016/j.powtec.2019.09.069.

Jin, Y.F., Yuan, W.H., Yin, Z.Y., Cheng, Y.M., 2020. An edge-based strain smoothing particle finite element method for large deformation problems in geotechnical engineering. International Journal for Numerical and Analytical Methods in Geomechanics 44, 923-941. doi:10.1002/nag.3016.

Jin, Z., Yin, Z.Y., Kotronis, P., Jin, Y.F., 2019a. Numerical investigation on evolving failure of caisson foundation in sand using the combined lagrangian-sph method. Marine Georesources \& Geotechnology 37, $23-35$.

Jin, Z., Yin, Z.Y., Kotronis, P., Li, Z., 2019b. Advanced numerical modelling of caisson foundations in sand to investigate the failure envelope in the hmv space. Ocean Engineering 190, 106394.

Kermani, E., Qiu, T., 2020. Simulation of quasi-static axisymmetric collapse of granular columns using smoothed particle hydrodynamics and discrete element methods. Acta Geotechnica 15, 423-437.

La Ragione, L., Prantil, V.C., Sharma, I., 2008. A simplified model for inelastic behavior of an idealized granular material. International Journal of Plasticity 24, 168-189.

Lajeunesse, E., Monnier, J., Homsy, G., 2005. Granular slumping on a horizontal surface. Physics of fluids $17,103302$.

Li, H., Deng, J., Yin, J., Zhu, J., 2020. Modelling the shearing behaviour of joints using an improved shear box genesis approach in particle flow code $(2 \mathrm{~d})$ and its validation. Geomechanics and Geophysics for Geo-Energy and Geo-Resources $6,1-15$.

Liang, W., Zhao, J., 2019. Multiscale modeling of large deformation in geomechanics. International Journal for Numerical and Analytical Methods in Geomechanics 43, 1080-1114.

Love, A.E.H., 2013. A treatise on the mathematical theory of elasticity. volume 1. Cambridge University Press.

Lube, G., Huppert, H.E., Sparks, R.S.J., Freundt, A., 2005. Collapses of two-dimensional granular columns. Physical Review E 72, 041301.

Lube, G., Huppert, H.E., Sparks, R.S.J., Hallworth, M.A., 2004. Axisymmetric collapses of granular columns. Journal of Fluid Mechanics 508, 175.

Lucy, L.B., 1977. A numerical approach to the testing of the fission hypothesis. The astronomical journal 82, $1013-1024$.

Mehrabadi, M.M., Nemat-Nasser, S., Oda, M., 1982. On statistical description of stress and fabric in granular materials. International Journal for Numerical and Analytical Methods in Geomechanics 6, 95-108.

Molenkamp, F., Nazemi, A.H., 2003. Interactions between two rough spheres, water bridge and water vapour. Géotechnique $53,255-264$

Monaghan, J.J., 1988. An introduction to sph. Comput. Phys. Comm. 48, 89-96.

Nguyen, C.T., Nguyen, C.T., Bui, H.H., Nguyen, G.D., Fukagawa, R., 2017. A new sph-based approach to simulation of granular flows using viscous damping and stress regularisation. Landslides 14, 69-81.

Nicot, F., Darve, F., 2011. The H-microdirectional model: accounting for a mesoscopic scale. Mechanics of Materials 43, 918-929.

Nicot, F., Darve, F., Group, R., 2005. A multi-scale approach to granular materials. Mechanics of materials 37, $980-1006$.

Peng, C., Wang, S., Wu, W., Yu, H.s., Wang, C., Chen, J.y., 2019. Loquat: an open-source gpu-accelerated sph solver for geotechnical modeling. Acta Geotechnica 14, 1269-1287. 
Peng, C., Wu, W., Yu, H.s., Wang, C., 2015. A sph approach for large deformation analysis with hypoplastic constitutive model. Acta Geotechnica 10, 703-717.

Phillips, J.C., Hogg, A.J., Kerswell, R.R., Thomas, N.H., 2006. Enhanced mobility of granular mixtures of fine and coarse particles. Earth and Planetary Science Letters 246, 466-480.

Rondon, L., Pouliquen, O., Aussillous, P., 2011. Granular collapse in a fluid: role of the initial volume fraction. Physics of Fluids 23, 073301.

Shreve, R.L., 1968. Leakage and fluidization in air-layer lubricated avalanches. Geological Society of America Bulletin 79, $653-658$.

Sołowski, W., Sloan, S., 2015. Evaluation of material point method for use in geotechnics. International Journal for Numerical and Analytical Methods in Geomechanics 39, 685-701.

Staron, L., Hinch, E., 2005. Study of the collapse of granular columns using two-dimensional discrete-grain simulation. Journal of Fluid Mechanics 545, 1-27.

Systèmes, D., 2014. Abaqus 6.14 analysis user manual.

Tan, D.Y., Yin, J.H., Feng, W.Q., Zhu, Z.H., Qin, J.Q., Chen, W.B., 2019. New simple method for calculating impact force on flexible barrier considering partial muddy debris flow passing through. Journal of Geotechnical and Geoenvironmental Engineering 145, 04019051.

Tan, D.Y., Yin, J.H., Qin, J.Q., Zhu, Z.H., Feng, W.Q., 2020. Experimental study on impact and deposition behaviours of multiple surges of channelized debris flow on a flexible barrier. Landslides , 1-13.

Utili, S., Zhao, T., Houlsby, G., 2015. 3d dem investigation of granular column collapse: evaluation of debris motion and its destructive power. Engineering geology 186, 3-16.

Wang, G., Riaz, A., Balachandran, B., 2019. Smooth particle hydrodynamics studies of wet granular column collapses. Acta Geotechnica , 1-13.

Wu, Z.X., Ji, H., Han, J., Yu, C., 2019. Numerical modelling of granular column collapse using coupled eulerian-lagrangian technique with critical state soil model. Engineering Computations .

Xiong, H., Nicot, F., Yin, Z., 2017. A three-dimensional micromechanically based model. International Journal for Numerical and Analytical Methods in Geomechanics 41, 1669-1686.

Xiong, H., Nicot, F., Yin, Z., 2019a. From micro scale to boundary value problem: using a micromechanically based model. Acta Geotechnica 14, 1307-1323.

Xiong, H., Yin, Z.Y., Nicot, F., 2019b. A multiscale work-analysis approach for geotechnical structures. International Journal for Numerical and Analytical Methods in Geomechanics 43, 1230-1250.

Xiong, H., Yin, Z.Y., Nicot, F., 2020. Programming a micro-mechanical model of granular materials in julia. Advances in Engineering Software 145, 102816.

Yin, Z.Y., Jin, Z., Kotronis, P., Wu, Z.X., 2018. Novel sph simsand-based approach for modeling of granular collapse. International Journal of Geomechanics 18, 04018156

Yuan, Y., Lee, T.R., 2013. Contact Angle and Wetting Properties. Springer Berlin Heidelberg, Berlin, Heidelberg. pp. $3-34$.

Zhang, X., Krabbenhoft, K., Sheng, D., Li, W., 2015. Numerical simulation of a flow-like landslide using the particle finite element method. Computational Mechanics 55, 167-177.

Zhao, C.F., Kruyt, N.P., 2020. An evolution law for fabric anisotropy and its application in micromechanical modelling of granular materials. International Journal of Solids and Structures

Zhao, C.F., Yin, Z.Y., Hicher, P.Y., 2018. Integrating a micromechanical model for multiscale analyses. International Journal for Numerical Methods in Engineering 114, 105-127.

Zhao, Y.R., Yang, H.Q., Huang, L.P., Chen, R., Chen, X.S., Liu, S.Y., 2019. Mechanical behavior of intact completely decomposed granite soils along multi-stage loading-unloading path. Engineering Geology 260, 105242. doi:10.1016/j. enggeo.2019.105242.

Zhu, H., Mehrabadi, M.M., Massoudi, M., 2006. Three-dimensional constitutive relations for granular materials based on the dilatant double shearing mechanism and the concept of fabric. International journal of plasticity $22,826-857$.

Zhu, Q., Shao, J.F., Mainguy, M., 2010. A micromechanics-based elastoplastic damage model for granular materials at low confining pressure. International Journal of Plasticity 26, 586-602. 